\title{
Heavy quarks within the electroweak multiplet
}

\author{
J. Besprosvany and R. Romero \\ Instituto de Física, Universidad Nacional Autónoma de México, \\ Apartado Postal 20-364, Ciudad de México 01000, México
}

(Received 1 December 2015; published 2 April 2019; corrected 1 May 2019)

\begin{abstract}
Standard model fields and their associated electroweak Lagrangian are equivalently expressed in a shared spin basis. The scalar-vector terms are written with scalar-operator components acting on quarkdoublet elements, and shown to be parametrization-invariant. Such terms, and the t- and b-quark Yukawa terms are linked by the identification of the common mass-generating Higgs operating upon the other fields, after acquiring a vacuum expectation value $v$. Thus, the customary vector masses are related to the fermions', fixing the t-quark mass $m_{t}$ with the relation $m_{t}^{2}+m_{b}^{2}=v^{2} / 2$, either for maximal hierarchy, or given the b-quark mass $m_{b}$. A sum rule is derived for all quark masses that generalizes this restriction. An interpretation follows that electroweak bosons and heavy quarks belong in a multiplet.
\end{abstract}

DOI: 10.1103/PhysRevD.99.073001

\section{INTRODUCTION}

The standard model (SM) describes elementary-particle features and their interactions, which is praiseworthy, given its relatively limited required input, consisting of specific gauge and flavor symmetries, representations, and parameters, yet aspects remain within the model whose origin and connection to other tenets is absent and that need to be addressed.

Thus, among its successes, the SM predicts mass values for the $\mathrm{W}$ and $\mathrm{Z}$ bosons [1] that carry the short-range electroweak interaction, in terms of electroweak parameters, through the Higgs mechanism [2,3]. However, one salient SM problem is that the fermion sector and its masses remain arbitrary, as they arise from Lagrangian terms, independent from the boson elements.

The electroweak sector hints that it may provide this link, given that the $\mathrm{W}$ and $\mathrm{Z}$ vectors have universal couplings to SM fermions, and the Higgs field collectively gives mass to fermions and bosons. In addition, the similar order of magnitude of the measured masses [4] of the $\mathrm{W}, \mathrm{Z}$, the recently discovered scalar excitation, associated with the Higgs [5], and the top quark (with the bottom quark's the next highest), suggests connections among them, and thus, a common energy scale. Furthermore, fermions occupy the spin- $1 / 2$ and fundamental representations of the Lorentz and scalar groups, respectively, as vector bosons belong to the adjoint

Published by the American Physical Society under the terms of the Creative Commons Attribution 4.0 International license. Further distribution of this work must maintain attribution to the author(s) and the published article's title, journal citation, and DOI. Funded by SCOAP . representation of each group, ${ }^{1,2}$ which implies bosons can be constructed in terms of fermions, suggesting composite structures and/or a common origin.

The above inspires looking for a formalism that takes account of discrete degrees of freedom (d.o.f.) in a single basis, including group representation properties, such as the fermion-boson fundamental-adjoint duality for the Lorentzscalar representations, and that describes the combined action of operators on fields.

A previously proposed SM extension [6], based on a shared extended spin space, with a matrix formalism, satisfies these requirements, as it replicates SM fields with their features, and matrix multiplication accounts for operator action on fields. This space contains a $(3+1)$ dimensional subspace and one beyond $3+1$, linked, respectively, to Lorentz and scalar d.o.f. [7]. At each dimension, a finite number of Lorentz-invariant partitions are generated with specific symmetries and representations, reproducing particular SM features, where the cases with dimension $5+1$ [8], $7+1$ [9], and $9+1$ [10] were studied.

In this connection, it is worth recalling that a basis or representation choice can be useful, even essential, in the description of a system and its dynamics. It may reveal otherwise-hidden connections between its components, and provide a simpler framework to understand physical properties. Such a basis may describe effective d.o.f. [11] accounting for collective interactions, allowing for a simpler near free-particle description, in a first

\footnotetext{
${ }^{1}$ As the Higgs occupies the $\mathrm{SU}_{L}(2)$ fundamental representation.

${ }^{2}$ For the Abelian hypercharge group $\mathrm{U}(1)_{Y}$, gauge invariance ensures boson-fermion quantum-number additivity.
} 
approximation. For example, nucleon and associated boson interactive configurations give a tractable account of nuclear-motion modes [12]. Within condensed matter and low-temperature superconducting systems, a residual attractive interaction related to phonons couples electrons into Cooper pairs [13], which propagate freely, and lead to frictionless currents. In an application of this theory to quantum field theory and elementary particles [14], a fourfermion interaction produces fermion and composite-boson masses, linking their values. The quark model [15] conceives mesons and baryons in terms of constituent (dressed) quarks.

Leaving aside the more speculative nature of the spin SM extension, but complementarily to it, in this paper, we use it as a basis to derive SM connections, and the fields' mass values in particular: SM heavy-fermion $(F)$, vector $(V)$, and scalar $(S)$ fields are equivalently expressed in terms of the obtained common basis [6] for both Lorentz and electroweak d.o.f., in turn, recasting their Lagrangian components $\mathcal{L}=\mathcal{L}_{F V}+\mathcal{L}_{S V}+\mathcal{L}_{S F}$; the identification of the scalar operator within the $\mathcal{L}_{S F}$ and $\mathcal{L}_{S V}$ vertices links univocally its defining (mass) parameters. Indeed, such universal electroweakly-invariant terms lead, under the Higgs mechanism, to a scalar whose lowest-energy condensate state pervades space, and generates particle masses through its vacuum expectation value $v$. Within the spin basis, this mechanism is similarly represented; as these fields shape elements on a matrix space, with a single associated scalar operator acting upon the others, their mass-generation property relates their coefficients.

Next, as we give the paper's organization, we sketch the argument in more detail. Section II reviews the applied spin-extended space for symmetry generators and states. The paper focuses then on the $(7+1)$-dimensional case that can describe the electroweak sector, and a quark doublet. For all sectors, $\mathcal{L}_{F V}, \mathcal{L}_{S V}, \mathcal{L}_{S F}$, the conventional and spin-space Lagrangian are equivalent, which is shown term-by-term in Appendixes A and B. Section III chooses one among two vector bases within $\mathcal{L}_{F V}$, in which vectors with chiral properties are adequate. Section IV writes $\mathcal{L}_{S V}$ equivalently with combinations of the scalars and their conjugates, with universal couplings to vectors, shown explicitly in Appendix B, and similarly for the spin-base representation, in which these two scalars induce a projection to flavor-doublet components (as $t, b$ quarks). Schematically, given the spin-space basis element $B_{f}$ for a field $f(x)$, we write $\mathcal{L}_{S V}$ in terms of $B_{S^{\prime}}$ containing these two scalar components, obtaining the vector mass squared within $\mathcal{L}_{F V}$ as $\left[B_{S^{\prime}}, B_{V}\right]^{\dagger}\left[B_{S^{\prime}}, B_{V}\right]$. In Sec. V, we show that the fermion masses within $\mathcal{L}_{S F}$ can be written $\left[B_{S}, B_{F}\right]$, where $B_{F}$ contains two terms with appropriate Yukawa coefficients. Within the spin-basis formalism, we derive that $B_{S^{\prime}}, B_{S}$ have the same operator structure; given their mass-giving nature, the identification of these operators and their coefficients translates a $v$-normalization restriction on
$B_{S^{\prime}}$ to $B_{S}$, implying a relation for the $\mathrm{t}$ and $\mathrm{b}$ quark masses. Section VI shows a procedure exists that generalizes consistently this relation to all quarks in terms of a sum rule for their masses, taking advantage of the chiral projection properties of the scalar field in the spin basis. In Sec. VII, we draw conclusions. We work in the classical framework afforded by the Lagrangian, and at tree-level, but also rely on a quantum-mechanical interpretation.

\section{SYMMETRY GENERATORS AND STATES IN SPIN-EXTENDED SPACE}

In the following, we introduce the spin basis and its main features, where more information may be found in previous treatments [7-10]. Mainly, it describes SM discrete d.o.f. in a single scheme, namely, for the Lorentz and scalar groups, and for both symmetry generators and state representations, using a common matrix space:

\section{A. Matrix space}

Such a space is rendered by a Clifford algebra $\mathcal{C}_{N}$, generated by a set of even- $N 2^{N / 2} \times 2^{N / 2}$ gamma matrices, obeying the defining property [16]

$$
\gamma_{\alpha} \gamma_{\beta}+\gamma_{\beta} \gamma_{\alpha}=2 g_{\alpha \beta}
$$

where $g_{\alpha \beta}$ is the metric tensor with signature $(+,-, \cdots,-)$ and $^{3} \alpha, \beta=0,1, \ldots 3,5, \ldots, N$, whose combinations produce a complex matrix-space with dimension $2^{N}$.

The gamma matrices have Hermiticity properties

$$
\begin{aligned}
& \gamma_{0}^{\dagger}=\gamma_{0}, \\
& \gamma_{\delta}^{\dagger}=-\gamma_{\delta} \quad \delta=1, \ldots 3,5, \ldots, N .
\end{aligned}
$$

\section{B. Operators and symmetry transformations}

The Lorentz generators and transformations acting on spinors have standard expressions in the 4-dimensional Clifford algebra $\mathcal{C}_{4}$, namely,

$$
\begin{gathered}
\sigma_{\mu \nu}=\frac{i}{2}\left[\gamma_{\mu}, \gamma_{\nu}\right] \quad \text { with } \mu, \nu=0, \ldots, 3, \\
S(\Lambda)=e^{-\frac{i}{4} \sigma_{\mu \nu} \omega^{\mu \nu}},
\end{gathered}
$$

with the $(3+1)$-dimensional gamma matrices $\gamma_{\mu}$ transforming as vectors, while the remaining $N-4$ gamma matrices $\gamma_{a}, a=5, \ldots, N$, and their products commuting with $\sigma_{\mu \nu}$, so they are indeed Lorentz scalars identified with generators of continuous symmetries, either gauge or global. Together with the four-dimensional pseudoscalar

$$
\tilde{\gamma}_{5} \equiv-i \gamma_{0} \gamma_{1} \gamma_{2} \gamma_{3}
$$

\footnotetext{
${ }^{3}$ Following standard practice, the label 4 is omitted.
} 
the scalars are accommodated in the unitary symmetry set

$\mathcal{S}_{N-4}=\frac{1}{2}\left(1+\tilde{\gamma}_{5}\right) \mathrm{U}\left(2^{(N-4) / 2}\right) \oplus \frac{1}{2}\left(1-\tilde{\gamma}_{5}\right) \mathrm{U}\left(2^{(N-4) / 2}\right)$,

where 1 stands for the $N$-dimensional identity matrix.

A projector operator $\mathcal{P}$, obtained from elements of $\mathcal{S}_{N-4}$, within a limited number partitions, is chosen to fit as closely the SM. The combined operator that acts on both the Lorentz generators $\mathcal{J}_{\mu \nu}=i\left(x_{\mu} \partial_{\nu}-x_{\nu} \partial_{\mu}\right)+\frac{1}{2} \sigma_{\mu \nu}$ and the $\mathcal{S}_{N-4}$ symmetry-operator space is likewise projected

$$
\begin{aligned}
\mathcal{J}_{\mu \nu}^{\prime} & =\mathcal{P} \mathcal{J}_{\mu \nu}=\mathcal{P}\left[i\left(x_{\mu} \partial_{\nu}-x_{\nu} \partial_{\mu}\right)+\frac{1}{2} \sigma_{\mu \nu}\right], \\
\mathcal{S}_{N-4}^{\prime} & =\mathcal{P} \mathcal{S}_{N-4} .
\end{aligned}
$$

Lorentz transformations are thus

$$
S(\Lambda)=e^{-\frac{i}{4} \mathcal{P} \omega^{\mu \nu} \sigma_{\mu \nu}}
$$

and scalar transformations have the form

$$
U=\exp \left[-i I_{a} \alpha_{a}(x)\right]
$$

with $I_{a} \in \mathcal{S}_{N-4}^{\prime}$. Symmetry generators within this space are described schematically in Fig. 1 in Ref. [9]. The inner product of two fields is defined according to a matrix space

$$
\langle\phi \mid \Psi\rangle=\operatorname{tr}\left(\phi^{\dagger} \Psi\right) .
$$

Under a unitary transformation, $\Psi \rightarrow U \Psi U^{\dagger}$, given the ket-bra matrix structure [7], with the bras interpreted as conjugate states. Thus, a Hermitian operator $O p$ within this space characterizes a state $\Psi$ with the eigenvalue rule

$$
[O p, \Psi]=\lambda \Psi,
$$

for real $\lambda$. This definition is consistent with the action of a derivative operator on a Hilbert space: $[\vec{\partial}, \Psi]=$ $[-\overleftarrow{\partial}, \Psi]=[\Psi, \overleftarrow{\partial}]$. The direct product $\operatorname{tr} \Psi_{b}^{\dagger} \Psi_{a}$ is also consistent associativity-wise with the operator rule, as $\operatorname{tr}\left[O p, \Psi_{b}\right]^{\dagger} \Psi_{a}=\operatorname{tr} \Psi_{b}^{\dagger}\left[O p^{\dagger}, \Psi_{a}\right]$.

\section{Field representation}

Fields are usually assumed to exist on a Cartesian basis; for example, a vector field has components $A_{\mu}(x)=g_{\mu}{ }^{\nu} A_{\nu}(x)$; alternatively, in the spin basis, it is expressed as $A_{\mu}(x)\left(\gamma_{0} \gamma^{\mu}\right)_{\alpha \beta}$ (the $\alpha \beta$ indices now specify the vector character.)

More generally, a physical field with scalar quantum numbers is associated with elements of $\mathcal{C}_{N}$, classified by operators from $\mathcal{C}_{4} \otimes \mathcal{S}_{N-4}$, so it has the structure (elements of $3+1$ space $) \times\left(\right.$ elements of $\left.\mathcal{S}_{N-4}\right)$.

Figure 2 in Ref. [9] shows the corresponding Lorentz states: scalars, vectors, fermions, and antisymmetric tensors, arranged in the same matrix space. Next, we provide more details on the first three (physical) fields.

\section{Fermion field}

When $\psi$ is a spin- $1 / 2$ particle, it may be seen schematically conformed as $\Psi \sim\left|\psi_{1}\right\rangle\left|a_{i} F_{1}\right\rangle\left\langle F_{2}\right|$, with the ket carrying spin-1/2 and gauge-group fundamental representation $\psi_{i}, a_{i}$ quantum numbers, respectively, and both the bra and ket carrying flavor group $F_{i}$.

More specifically, a fermion can have the form

$$
\psi_{\alpha}^{a}(x) L^{\alpha} P_{F} \Gamma_{a}^{F},
$$

where $\Gamma_{a}^{F}$ is an element of $\mathcal{S}_{N-4}^{\prime}$, and $L^{\alpha}$ represents a spin polarization component, e.g., $L^{1}=\left(\gamma_{1}+i \gamma_{2}\right)$. The operator $P_{F}$ is a projection operator, e. g., $P_{F}=L_{5}$, where

$$
R_{5}=\frac{1}{2}\left(1+\tilde{\gamma}_{5}\right), \quad L_{5}=\frac{1}{2}\left(1-\tilde{\gamma}_{5}\right),
$$

implying

$$
P_{F} \gamma^{\mu}=\gamma^{\mu} P_{F}^{c},
$$

with $P_{F}^{c}=1-P_{F}$, so that Lorentz and gauge generators act trivially on its rhs when evaluating commutators as in Eq. (11), since $P_{F}^{c} P_{F}=\left(1-P_{F}\right) P_{F}=0$.

Thus, for $U$ accounting for the Lorentz representation in Eq. (8) and the scalar transformation in Eq. (9), $\Psi$ transforms, unlike vector and scalar fields, as

$$
\Psi \rightarrow U \Psi
$$

This leads to fermions transforming as the fundamental representation of both the Lorentz and gauge groups.

\section{Vector field}

We may view vectors constructed as $\Psi \sim$ $\left|\psi_{1}\right\rangle\left|a_{1}\right\rangle\left\langle a_{2}\right|\left\langle\psi_{2}\right|$, with the bra-ket configuration producing Lorentz vector and gauge group adjoint configurations, given the vector and scalar $\gamma^{\mu}, \mu=0, \ldots, 3$ and $\gamma^{a}$, $a=5, \ldots, N$, respective transformation properties. Thus a vector field has form

$$
A_{\mu}^{a}(x) \gamma_{0} \gamma_{\mu} I_{a}
$$

where $\gamma_{0} \gamma_{\mu} \in \mathcal{C}_{4}$ and $I_{a} \in \mathcal{S}_{N-4}^{\prime}$ is a generator of a given unitary group. 


\section{Scalar field}

$\Psi \sim\left|\psi_{1}\right\rangle\left|a_{1}\right\rangle\left\langle a_{2}\right|\left\langle\psi_{2}\right|$, with the bra-ket configuration producing Lorentz scalar and gauge group fundamental configurations. In this case, a ket contains right-handed and a bra left-handed spin-1/2 components (or vice versa), reproducing the mass term and Higgs quantum numbers.

$$
\phi^{a}(x) \gamma_{0} \Gamma_{a}^{S},
$$

with $\Gamma_{a}^{S}$ an element of $\mathcal{S}_{N-4}^{\prime}$.

\section{Lagrangian formulation}

Interactive Lagrangians [7] can be given in terms of vector, scalar and fermion fields conforming to the general structure of operator action as in Eq. (11) and the inner product in Eq. (10). For example, a gauge-invariant fermion-vector Lagrangian is given by

$$
\frac{1}{N_{f}} \operatorname{tr} \Psi \dagger\left\{\left[i \partial_{\mu}-g A_{\mu}^{a}(x) I_{a}\right] \gamma^{0} \gamma^{\mu}-M \gamma^{0}\right\} \Psi,
$$

where $\Psi$ is a fermion field as in Eq. (13), $g$ is the coupling constant, $M$ is an appropriate mass operator, and $N_{f}$ contains the normalization. In the next subsection, we address the spin model in $7+1$ dimensions in connection with the SM, and whose basis states will allow to write $\mathcal{L}_{F V}, \mathcal{L}_{S V}, \mathcal{L}_{S F}$ in the next sections.

\section{E. $(7+1)$-dimensional model}

We next make a brief description of resulting states in a $(7+1)$-dimensional spin space under a useful partition for the SM description, sketching the way to obtain it, and providing graphic description.

\section{Operators}

The Clifford algebra is generated by eight $16 \times 16$ matrices

$$
\gamma_{0}, \gamma_{1}, \ldots, \gamma_{8}
$$

The matrices $\gamma^{0}, \gamma^{i}, i=1,2,3$ correspond to the Lorentz generators $\sigma_{\mu \nu}$, given in general in Eq. (3) and the remaining four matrices, together with all their different products, comprise the set $\mathcal{S}_{N-4}$ of scalars, with a cardinality of 32 . This set is, from Eq. (6), $\mathcal{S}_{4}=$ $P_{+} \mathrm{U}(4) \oplus P_{-} \mathrm{U}(4)$, with $P_{ \pm}=\frac{1}{2}\left(1 \pm \tilde{\gamma}_{5}\right), 1$ the $16 \times 16$ identity matrix and $\tilde{\gamma}_{5}$ the four-dimensional chirality matrix. The elements of $\mathrm{U}(4)$ consist of four matrices $\gamma_{a}, a=5, \ldots, 8$, six pairs $\gamma_{a b} \equiv \gamma_{a} \gamma_{b}, a<b$, four triplets $\gamma_{a b c} \equiv \gamma_{a} \gamma_{b} \gamma_{c}$, and one quadruplet $\gamma_{5} \gamma_{6} \gamma_{7} \gamma_{8}$. The Cartan subalgebra $\mathfrak{h}$ of $\mathcal{S}_{N-4}$ contains eight elements, and a suitable choice is given by
$1, \tilde{\gamma}_{5}, \gamma_{5} \gamma_{6}, \gamma_{7} \gamma_{8}, \gamma_{5} \gamma_{6} \gamma_{7} \gamma_{8}, \gamma_{5} \gamma_{6} \tilde{\gamma}_{5}, \gamma_{7} \gamma_{8} \tilde{\gamma}_{5}, \gamma_{5} \gamma_{6} \gamma_{7} \gamma_{8} \tilde{\gamma}_{5}$.

Since $\mathfrak{h}$ is conformed of all simultaneously diagonalizable operators, it is convenient to recast this basis in terms of the projection operators

$$
\begin{aligned}
& P_{R 1}=\frac{1}{8}\left(1+\tilde{\gamma}_{5}\right)\left(1+i \gamma_{5} \gamma_{6}\right)\left(1+i \gamma_{7} \gamma_{8}\right), \\
& P_{R 2}=\frac{1}{8}\left(1+\tilde{\gamma}_{5}\right)\left(1+i \gamma_{5} \gamma_{6}\right)\left(1-i \gamma_{7} \gamma_{8}\right), \\
& P_{R 3}=\frac{1}{8}\left(1+\tilde{\gamma}_{5}\right)\left(1-i \gamma_{5} \gamma_{6}\right)\left(1+i \gamma_{7} \gamma_{8}\right), \\
& P_{R 4}=\frac{1}{8}\left(1+\tilde{\gamma}_{5}\right)\left(1-i \gamma_{5} \gamma_{6}\right)\left(1-i \gamma_{7} \gamma_{8}\right), \\
& P_{L 1}=\frac{1}{8}\left(1-\tilde{\gamma}_{5}\right)\left(1+i \gamma_{5} \gamma_{6}\right)\left(1+i \gamma_{7} \gamma_{8}\right), \\
& P_{L 2}=\frac{1}{8}\left(1-\tilde{\gamma}_{5}\right)\left(1+i \gamma_{5} \gamma_{6}\right)\left(1-i \gamma_{7} \gamma_{8}\right), \\
& P_{L 3}=\frac{1}{8}\left(1-\tilde{\gamma}_{5}\right)\left(1-i \gamma_{5} \gamma_{6}\right)\left(1+i \gamma_{7} \gamma_{8}\right), \\
& P_{L 4}=\frac{1}{8}\left(1-\tilde{\gamma}_{5}\right)\left(1-i \gamma_{5} \gamma_{6}\right)\left(1-i \gamma_{7} \gamma_{8}\right),
\end{aligned}
$$

which run along the diagonal in the matrix space (Fig. 1).

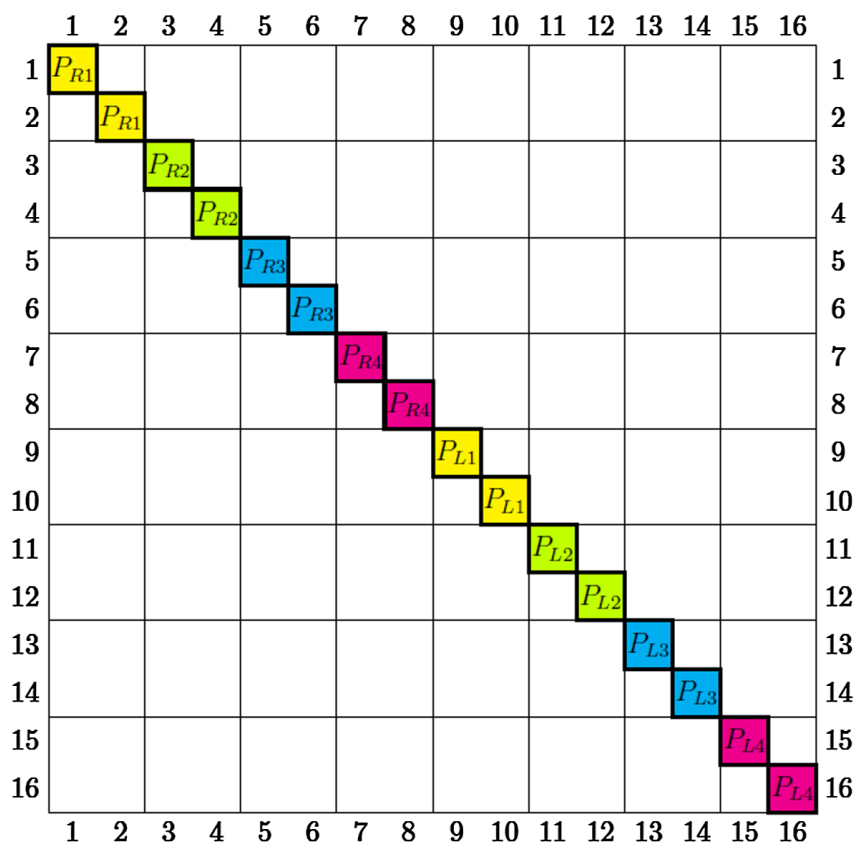

FIG. 1. Matrix representation of the Cartan basis [cf. Eq. (21)] in extended spin space in $7+1$ dimensions. The eightdimensional basis is represented here in terms of the projection operators $P_{R, L i}, i=1, \ldots, 4$. The subscripts $R, L$ refer to the chirality: $R$ for operators containing $1+\tilde{\gamma}_{5}$ (right-handed), and $L$ for operators containing $1-\tilde{\gamma}_{5}$ (left-handed). 
The operators that classify the states, with examples in terms of the projectors, consist of the baryon-number operator

$B=\frac{1}{6}\left(1-i \gamma_{5} \gamma_{6}\right)=\frac{1}{3}\left(P_{R 3}+P_{R 4}+P_{L 3}+P_{L 4}\right)$,

the $\mathrm{U}(1)$ hypercharge generator

$$
\begin{aligned}
Y_{o} & =\frac{1}{3}\left(4 P_{R 3}-2 P_{R 4}+P_{L 3}+P_{L 4}\right), \\
& =\frac{1}{6}\left(1-i \gamma_{5} \gamma_{6}\right)\left(1+i \frac{3}{2}\left(1+\tilde{\gamma}_{5}\right) \gamma_{7} \gamma_{8}\right),
\end{aligned}
$$

and $I_{3}$ within the $\mathrm{SU}(2)$ weak isospin generators

$$
\begin{aligned}
I_{1} & =\frac{i}{8}\left(1-\tilde{\gamma}_{5}\right)\left(1-i \gamma_{5} \gamma_{6}\right) \gamma^{7}, \\
I_{2} & =\frac{i}{8}\left(1-\tilde{\gamma}_{5}\right)\left(1-i \gamma_{5} \gamma_{6}\right) \gamma^{8}, \\
I_{3} & =\frac{1}{2}\left(P_{L 3}-P_{L 4}\right)=\frac{i}{8}\left(1-\tilde{\gamma}_{5}\right)\left(1-i \gamma_{5} \gamma_{6}\right) \gamma_{7} \gamma_{8} .
\end{aligned}
$$

The charge operator is defined in the standard way by the Gell-Mann-Nishijima relation

$$
Q=I_{3}+\frac{Y_{o}}{2}
$$

There are also flavor operators, forming the groups $\mathrm{SU}(2)_{f}, \mathrm{SU}(2)_{\hat{f}}, \mathrm{U}(1)_{f}$, and $\mathrm{U}(1)_{\hat{f}}$, and given by

$$
\begin{aligned}
& f_{1}=\frac{i}{8}\left(1+\tilde{\gamma}_{5}\right)\left(1+i \gamma^{5} \gamma^{6}\right) \gamma^{7}, \\
& f_{2}=\frac{i}{8}\left(1+\tilde{\gamma}_{5}\right)\left(1+i \gamma^{5} \gamma^{6}\right) \gamma^{8}, \\
& f_{3}=\frac{i}{8}\left(1+\tilde{\gamma}_{5}\right)\left(1+i \gamma^{5} \gamma^{6}\right) \gamma^{7} \gamma^{8}, \\
& \hat{f}_{1}=\frac{i}{8}\left(1-\tilde{\gamma}_{5}\right)\left(1+i \gamma^{5} \gamma^{6}\right) \gamma^{7}, \\
& \hat{f}_{2}=\frac{i}{8}\left(1-\tilde{\gamma}_{5}\right)\left(1+i \gamma^{5} \gamma^{6}\right) \gamma^{8}, \\
& \hat{f}_{3}=\frac{i}{8}\left(1-\tilde{\gamma}_{5}\right)\left(1+i \gamma^{5} \gamma^{6}\right) \gamma^{7} \gamma^{8},
\end{aligned}
$$

respectively, for $\mathrm{SU}(2)_{f}$ and $\mathrm{SU}(2)_{\hat{f}}$, and

$$
\begin{gathered}
f_{0}=i \gamma^{5} \gamma^{6} \tilde{\gamma}_{5}, \\
\hat{f}_{0}=i \gamma^{5} \gamma^{6},
\end{gathered}
$$

for $\mathrm{U}(1)_{f}$, and $\mathrm{U}(1)_{\hat{f}}$. The operators $f_{3}, \hat{f}_{3}, f_{0}$ and $\hat{f}_{0}$ belong to $\mathfrak{h}$. In Fig. 1, the matrix space is represented schematically. The diagonal operators classify the states (off-diagonal) acting from the left for states in the same row, and from the right for states in the same column, which is consistent with matrix multiplication. We also define a combination of diagonal flavor operators that classify states, given by

$$
\hat{F}=-\frac{1}{4}\left(\hat{f}_{0}-4 \hat{f}_{3}-8 f_{3}\right)
$$

\section{States}

States contain scalars, fermions, and vectors. Only the first two are considered in this section. The matrix space admits two Higgs doublets $\boldsymbol{\phi}_{1}$ and $\boldsymbol{\phi}_{2}$ (Table I and Fig. 2). They satisfy $\boldsymbol{\phi}_{1}=\tilde{\gamma}_{5} \boldsymbol{\phi}_{2}$. Their connection to Hermitian and SU(2) conjugates is clarified in Sec. IV C.

Non-Higgs scalars can also be constructed that contribute to the diagonalization of massive states. Reference [9] provides further information on their nature and their application to obtain fermion properties.

The massless-fermion states satisfy the general structure of Eq. (12) and have massless quark quantum numbers, when classified by baryon number, isospin, and

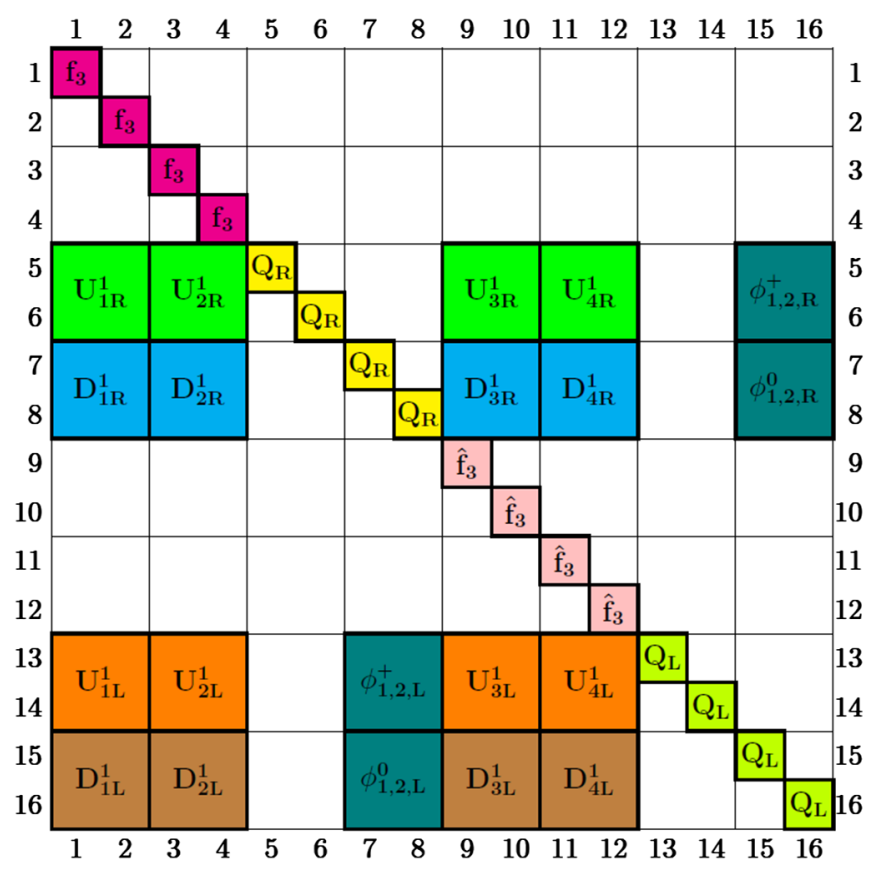

FIG. 2. Matrix representation of operators, massless quarks $\left(U_{i L, R}^{1}, D_{i L, R}^{1}, i=1, \ldots, 4\right)$ and Higgs $\left(\phi_{1,2}^{+}, \phi_{1,2}^{0}\right)$ d.o.f. in $(7+1)-$ dimensional spin space. The chiral projections of the diagonal operators $B, I_{3}$ and $Y_{o}$ are grouped together and represented by the sets $\mathbf{Q}_{R}=\frac{1}{2}\left(1+\tilde{\gamma}_{5}\right)\left(B, I_{3}, Y_{o}\right)$ and $\mathbf{Q}_{L}=\frac{1}{2}\left(1-\tilde{\gamma}_{5}\right)\left(B, Y_{o}\right)$. Following matrix multiplication rules, operators act from the left on states in the same row, and from the right on states in the same column. 
TABLE I. Scalar Higgs-like doublets.

\begin{tabular}{lccc}
\hline \hline Baryon number zero, Higgs-like scalars & $I_{3}$ & $Y_{o}$ & $Q$ \\
\hline$\phi_{1}=\left(\begin{array}{c}\phi_{1}^{+} \\
\phi_{1}^{0}\end{array}\right)=\left(\begin{array}{c}\frac{1}{8}\left(1-i \gamma^{5} \gamma^{6}\right)\left(\gamma^{7}+i \gamma^{8}\right) \gamma_{0} \\
\frac{1}{8}\left(1-i \gamma^{5} \gamma^{6}\right)\left(1+i \gamma^{7} \gamma^{8} \tilde{\gamma}_{5}\right) \gamma_{0}\end{array}\right)$ & $1 / 2$ & 1 & 1 \\
$\phi_{2}=\left(\begin{array}{c}\phi_{2}^{+} \\
\phi_{2}^{0}\end{array}\right)=\left(\begin{array}{c}\frac{1}{8}\left(1-i \gamma^{5} \gamma^{6}\right)\left(\gamma^{7}+i \gamma^{8}\right) \tilde{\gamma}_{5} \gamma_{0} \\
\frac{i}{8}\left(1-i \gamma^{5} \gamma^{6}\right)\left(1+i \gamma^{7} \gamma^{8} \tilde{\gamma}_{5}\right) \gamma^{7} \gamma^{8} \gamma_{0}\end{array}\right)$ & $-1 / 2$ & & 0 \\
\hline \hline
\end{tabular}

hypercharge. The matrix space admits four generations of quarks of different flavor (Fig. 2), arranged in four $\mathrm{SU}(2)_{L}$ doublets and eight right-handed singlets, shown in Tables II and III, respectively. After electroweak symmetry breaking, the Higgs generates a mass operator used in Sec. V to obtain fermion mass states.

\section{F. Fermion Yukawa elements}

Bilinear fermion terms can be constructed that produce scalar elements transforming quarks into their different combinations. We use the $(7+1)$-dimensional space represented in Fig. 2, with particular and general properties that can be distinguished.

There are two matrix configurations:

$$
P_{i}^{F \alpha \beta}=Q_{i R}^{\alpha} \bar{Q}_{i L}^{\beta} \quad i=1,2,3
$$

is contained in the Dirac projector with $(\alpha, \beta)$-spin components and (positive or negative)-energy; the three $P_{i}^{F \alpha \beta}$ are the same up to a phase; $Q$ are $U$ - or $D$-type fermions obtained from Tables II and III, defining $F$, the $R$, $L$ case taken as an example, and $\bar{Q}_{i L}^{\alpha}=Q_{i L}^{\alpha}{ }^{\dagger} \gamma_{0}^{B}$;

$$
\gamma_{0}^{B}=2\left(\phi_{1}^{0}+\phi_{1}^{0 \dagger}\right)
$$

with $\phi_{1}^{0}$ defined in Table I. The $i, j$ imply we choose a threegeneration (arbitrary) projection to reproduce the SM; we also note that $Q_{i R}^{\alpha} \bar{Q}_{j L}^{\beta}=0$ for $i \neq j$.

On the other hand,

$$
Y_{i j}^{F}=\bar{Q}_{i R}^{\alpha} Q_{j L}^{\alpha} \quad i, j=1,2,3
$$

defines the Yukawa basis (full flavor transition matrix) to be used in Sec. VI, for the complete scalar-fermion SM Lagrangian component. One can check that $Y_{i j}^{U}, Y_{i j}^{D}$ are the same (up to phases), so they are commonly labelled $Y_{i j}^{F}$. The set $R, L, \alpha$ is arbitrary and other choices will reproduce (up to phases) the nine $Y_{i j}^{F}$ terms. Indeed, although the $(7+1)$-dimensional basis can accommodate four generations, the projection operator for, say, flavors $1,2,3$

$$
Y^{F 4}=Y_{11}^{F}+Y_{22}^{F}+Y_{33}^{F},
$$

induces the three-generation subset with nine elements, $Y^{F 4 \dagger} Y_{i j}^{F} Y^{F 4}$. As the set is closed under matrix multiplication, the fourth generation is discarded (see Sec. VI.)

The resulting projection operators may be understood from the products of a fermion with matrix structure $\mid$ spin $\rangle\langle$ flavor $|$ and an Hermitian conjugate one, resulting in the form $|\operatorname{spin}\rangle\langle$ spin $|$ for Eq. (32) and, inverting the order, $\mid$ flavor $\rangle\langle$ flavor $|$ in Eq. (34).

\begin{tabular}{|c|c|c|c|c|c|}
\hline $\begin{array}{l}\text { Baryon number } 1 / 3 \text {, hypercharge } 1 / 3 \text { and polarization } 1 / 2 \text { (operator } \frac{3}{2} i B \gamma^{1} \gamma^{2} \text { ), } \\
\text { left-handed quark doublets }\end{array}$ & $I_{3}$ & $Q$ & $f_{3}$ & $\hat{f}_{3}$ & $F$ \\
\hline $\mathbf{Q}_{1 L}^{1}=\left(\begin{array}{c}U_{1 L}^{1} \\
D_{1 L}^{1}\end{array}\right)=\left(\begin{array}{c}\frac{1}{16}\left(1-\tilde{\gamma}_{5}\right)\left(\gamma^{5}-i \gamma^{6}\right)\left(\gamma^{7}+i \gamma^{8}\right)\left(\gamma^{0}+\gamma^{3}\right) \\
\frac{1}{16}\left(1-\tilde{\gamma}_{5}\right)\left(\gamma^{5}-i \gamma^{6}\right)\left(1-i \gamma^{7} \gamma^{8}\right)\left(\gamma^{0}+\gamma^{3}\right)\end{array}\right)$ & $\begin{array}{c}1 / 2 \\
-1 / 2\end{array}$ & $\begin{array}{l}2 / 3 \\
-1 / 3\end{array}$ & $\begin{array}{l}1 / 2 \\
1 / 2\end{array}$ & 0 & $\begin{array}{l}3 / 2 \\
3 / 2\end{array}$ \\
\hline $\mathbf{Q}_{2 L}^{1}=\left(\begin{array}{c}U_{2 L}^{1} \\
D_{2 L}^{1}\end{array}\right)=\left(\begin{array}{c}\frac{1}{16}\left(1-\tilde{\gamma}_{5}\right)\left(\gamma^{5}-i \gamma^{6}\right)\left(1+i \gamma^{7} \gamma^{8}\right)\left(\gamma^{0}+\gamma^{3}\right) \\
\frac{1}{16}\left(1-\tilde{\gamma}_{5}\right)\left(\gamma^{5}-i \gamma^{6}\right)\left(\gamma^{7}-i \gamma^{8}\right)\left(\gamma^{0}+\gamma^{3}\right)\end{array}\right)$ & $\begin{array}{c}1 / 2 \\
-1 / 2\end{array}$ & $\begin{array}{c}2 / 3 \\
-1 / 3\end{array}$ & $\begin{array}{l}-1 / 2 \\
-1 / 2\end{array}$ & 0 & $\begin{array}{l}-1 / 2 \\
-1 / 2\end{array}$ \\
\hline $\mathbf{Q}_{3 L}^{1}=\left(\begin{array}{c}U_{3 L}^{1} \\
D_{3 L}^{1}\end{array}\right)=\left(\begin{array}{c}\frac{1}{16}\left(1-\tilde{\gamma}_{5}\right)\left(\gamma^{5}-i \gamma^{6}\right)\left(\gamma^{7}+i \gamma^{8}\right) \gamma^{0}\left(\gamma^{0}-\gamma^{3}\right) \\
\frac{1}{16}\left(1-\tilde{\gamma}_{5}\right)\left(\gamma^{5}-i \gamma^{6}\right)\left(1-i \gamma^{7} \gamma^{8}\right) \gamma^{0}\left(\gamma^{0}-\gamma^{3}\right)\end{array}\right)$ & $\begin{array}{c}1 / 2 \\
-1 / 2\end{array}$ & $\begin{array}{l}2 / 3 \\
-1 / 3\end{array}$ & 0 & $\begin{array}{l}1 / 2 \\
1 / 2\end{array}$ & $\begin{array}{l}1 \\
1\end{array}$ \\
\hline $\mathbf{Q}_{4 L}^{1}=\left(\begin{array}{c}U_{4 L}^{1} \\
D_{4 L}^{1}\end{array}\right)=\left(\begin{array}{c}\frac{1}{16}\left(1-\tilde{\gamma}_{5}\right)\left(\gamma^{5}-i \gamma^{6}\right)\left(1+i \gamma^{7} \gamma^{8}\right) \gamma^{0}\left(\gamma^{0}-\gamma^{3}\right) \\
\frac{1}{16}\left(1-\tilde{\gamma}_{5}\right)\left(\gamma^{5}-i \gamma^{6}\right)\left(\gamma^{7}-i \gamma^{8}\right) \gamma^{0}\left(\gamma^{0}-\gamma^{3}\right)\end{array}\right)$ & $\begin{array}{c}1 / 2 \\
-1 / 2 \\
\end{array}$ & $\begin{array}{l}2 / 3 \\
-1 / 3 \\
\end{array}$ & 0 & $\begin{array}{l}-1 / 2 \\
-1 / 2 \\
\end{array}$ & 0 \\
\hline
\end{tabular}

TABLE II. Massless left-handed quark weak isospin doublets. Gauge and Lorentz operators act from the left and trivially from the right. To obtain the $-1 / 2$ polarization, the replacement must be made $\left(\gamma^{0}+\gamma^{3}\right) \rightarrow\left(\gamma^{1}-i \gamma^{2}\right)$, for $\mathbf{Q}_{1 L}^{1}, \mathbf{Q}_{2 L}^{1}$, and $\left(\gamma^{0}-\gamma^{3}\right) \rightarrow\left(\gamma^{1}-i \gamma^{2}\right)$, for $\mathbf{Q}_{3 L}^{1}, \mathbf{Q}_{4 L}^{1}$. 
TABLE III. Massless right-handed quark weak isospin singlets. Gauge and Lorentz operators act from the left and trivially from the right. To obtain the $-1 / 2$ polarization, the replacement must be made $\left(\gamma^{0}+\gamma^{3}\right) \rightarrow\left(\gamma^{1}-i \gamma^{2}\right)$, for $U_{1 R}^{1}, U_{2 R}^{1}, D_{1 R}^{1}, D_{2 R}^{1}$, and $\left(\gamma^{0}-\gamma^{3}\right) \rightarrow\left(\gamma^{1}-i \gamma^{2}\right)$, for $U_{3 R}^{1}, U_{4 R}^{1}, D_{3 R}^{1}, D_{4 R}^{1}$.

\begin{tabular}{|c|c|c|c|c|c|}
\hline $\begin{array}{l}\text { Baryon number } 1 / 3 \text { and polarization } 1 / 2 \text { (operator } \frac{3}{2} i B \gamma^{1} \gamma^{2} \text { ), } \\
\text { right-handed quark singlets }\end{array}$ & $Y_{o}$ & $Q$ & $f_{3}$ & $\hat{f}_{3}$ & $F$ \\
\hline$U_{1 R}^{1}=\frac{1}{16}\left(1+\tilde{\gamma}_{5}\right)\left(\gamma^{5}-i \gamma^{6}\right)\left(\gamma^{7}+i \gamma^{8}\right) \gamma^{0}\left(\gamma^{0}+\gamma^{3}\right)$ & $4 / 3$ & $2 / 3$ & $1 / 2$ & \multirow{2}{*}{0} & $3 / 2$ \\
\hline$D_{1 R}^{1}=\frac{1}{16}\left(1+\tilde{\gamma}_{5}\right)\left(\gamma^{5}-i \gamma^{6}\right)\left(1-i \gamma^{7} \gamma^{8}\right) \gamma^{0}\left(\gamma^{0}+\gamma^{3}\right)$ & $-2 / 3$ & $-1 / 3$ & $1 / 2$ & & $3 / 2$ \\
\hline$U_{2 R}^{1}=\frac{1}{16}\left(1+\tilde{\gamma}_{5}\right)\left(\gamma^{5}-i \gamma^{6}\right)\left(1+i \gamma^{7} \gamma^{8}\right) \gamma^{0}\left(\gamma^{0}+\gamma^{3}\right)$ & $4 / 3$ & $2 / 3$ & $-1 / 2$ & \multirow{2}{*}{0} & $-1 / 2$ \\
\hline$D_{2 R}^{1}=\frac{1}{16}\left(1+\tilde{\gamma}_{5}\right)\left(\gamma^{5}-i \gamma^{6}\right)\left(\gamma^{7}-i \gamma^{8}\right) \gamma^{0}\left(\gamma^{0}+\gamma^{3}\right)$ & $-2 / 3$ & $-1 / 3$ & $-1 / 2$ & & $-1 / 2$ \\
\hline$U_{3 R}^{1}=\frac{1}{16}\left(1+\tilde{\gamma}_{5}\right)\left(\gamma^{5}-i \gamma^{6}\right)\left(\gamma^{7}+i \gamma^{8}\right)\left(\gamma^{0}-\gamma^{3}\right)$ & $4 / 3$ & $2 / 3$ & \multirow{2}{*}{0} & $1 / 2$ & 1 \\
\hline$D_{3 R}^{1}=\frac{1}{16}\left(1+\tilde{\gamma}_{5}\right)\left(\gamma^{5}-i \gamma^{6}\right)\left(1-i \gamma^{7} \gamma^{8}\right)\left(\gamma^{0}-\gamma^{3}\right)$ & $-2 / 3$ & $-1 / 3$ & & $1 / 2$ & 1 \\
\hline$U_{4 R}^{1}=\frac{1}{16}\left(1+\tilde{\gamma}_{5}\right)\left(\gamma^{5}-i \gamma^{6}\right)\left(1+i \gamma^{7} \gamma^{8}\right)\left(\gamma^{0}-\gamma^{3}\right)$ & $4 / 3$ & $2 / 3$ & \multirow{2}{*}{0} & $-1 / 2$ & \multirow{2}{*}{0} \\
\hline$D_{4 R}^{1}=\frac{1}{16}\left(1+\tilde{\gamma}_{5}\right)\left(\gamma^{5}-i \gamma^{6}\right)\left(\gamma^{7}-i \gamma^{8}\right)\left(\gamma^{0}-\gamma^{3}\right)$ & $-2 / 3$ & $-1 / 3$ & & $-1 / 2$ & \\
\hline
\end{tabular}

\section{FERMION-VECTOR LAGRANGIAN: CHIRAL BASIS IN SPIN SPACE}

Concentrating on the heaviest fermions, the SM twoquark $^{4}$ electroweak interaction Lagrangian [1] is ${ }^{5}$

$$
\begin{aligned}
\mathcal{L}_{F V}= & \overline{\mathbf{q}}_{L}(x)\left[i \partial_{\mu}+\frac{1}{2} g \tau^{a} W_{\mu}^{a}(x)+\frac{1}{6} g^{\prime} B_{\mu}(x)\right] \gamma^{\mu} \mathbf{q}_{L}(x) \\
& +\bar{t}_{R}(x)\left[i \partial_{\mu}+\frac{2}{3} g^{\prime} B_{\mu}(x)\right] \gamma^{\mu} t_{R}(x) \\
& +\bar{b}_{R}(x)\left[i \partial_{\mu}-\frac{1}{3} g^{\prime} B_{\mu}(x)\right] \gamma^{\mu} b_{R}(x)
\end{aligned}
$$

where the spin- $1 / 2$ fields consist of $\mathbf{q}_{L}(x)=\left(\begin{array}{c}t_{L}(x) \\ b_{L}(x)\end{array}\right)$, a lefthanded hypercharge $Y=1 / 3 \mathrm{SU}(2)_{L}$-doublet, and $t_{R}(x)$, $b_{R}(x)$, right-handed $Y=4 / 3,-2 / 3$ singlets, respectively; each term contains two polarizations as, e.g., $t_{L}(x)=$ $\left(\begin{array}{l}\psi_{t L}^{1}(x) \\ \psi_{t L}^{2}(x)\end{array}\right) ; \psi_{q h}^{\alpha}(x)$ are wave functions ${ }^{6}$ for quarks $q=t, b$, with spin components $\alpha=1,2$, and chirality $h=L, R$; $W_{\mu}^{a}(x), a=1,2,3$, and $B_{\mu}(x)$, are associated gauge-group weak and hypercharge vector bosons, with coupling constants, $g, g^{\prime}$, respectively; $\tau^{a}$ are the Pauli matrices representing the $\mathrm{SU}(2)_{L}$ generators.

An extended $(7+1)$-dimensional Clifford algebra comprises a sufficiently large space to describe heavy SM particles [7,9], with the 4-dimensional Lorentz symmetry

\footnotetext{
${ }^{4} \mathrm{~A}$ single generation is used, and CKM mixing is neglected; Eq. (36) describes the electroweak interaction for one quark color, and a sum is assumed over each such term.

${ }^{5}$ We use units with $\hbar=c=1$, and metric $g_{\mu \nu}=(1,-1,-1,-1)$ throughout.

${ }^{6}$ For simplicity, spin and scalar representations are assumed that give the states' form.
}

maintained, and spin-component generators $\frac{3}{2} B \sigma_{\mu \nu}$, where $\sigma_{\mu \nu}=\frac{i}{2}\left[\gamma_{\mu}, \gamma_{\nu}\right]$, and $\mu, \nu=0, \ldots, 3$; additional scalar generators use $\gamma_{5}, \ldots, \gamma_{8}$, producing the baryon-number operator $B$ in Eq. (23), which conforms a spin-space projection partition, and gives quarks $1 / 3$ ( $-1 / 3$ for antiparticles,) and bosons 0 .

Other scalar-symmetry generators include the hypercharge $Y_{o}$ in Eq. (24), with $\tilde{\gamma}_{5}=-i \gamma_{0} \gamma_{1} \gamma_{2} \gamma_{3}$, the weak $\mathrm{SU}(2)_{L}$ terms in Eq. (25) and flavor generators in Eqs. (27)-(30); as required, $\left[I_{i}, I_{j}\right]=i \epsilon_{i j k} I_{k},\left[I_{i}, Y_{o}\right]=$ $\left[B, Y_{o}\right]=\left[B, I_{i}\right]=\left[3 B \sigma_{\mu \nu}, Y_{o}\right]=\left[3 B \sigma_{\mu \nu}, I_{i}\right]=0$.

The $(7+1)$-dimensional space allows for a description of quark fields,

$$
\begin{aligned}
\Psi_{q L}(x) & =\sum_{\alpha} \psi_{t L}^{\alpha}(x) T_{L}^{\alpha}+\psi_{b L}^{\alpha}(x) B_{L}^{\alpha}, \\
\Psi_{t R}(x) & =\sum_{\alpha} \psi_{t R}^{\alpha}(x) T_{R}^{\alpha}, \\
\Psi_{b R}(x) & =\sum_{\alpha} \psi_{b R}^{\alpha}(x) B_{R}^{\alpha},
\end{aligned}
$$

with hypercharges $1 / 3,4 / 3,-2 / 3$, respectively, and spinor components chosen in Table IV, given explicitly in Tables II and III; the quantum numbers $\lambda$ are obtained from the operator structure $[O p, \Psi]=\lambda \Psi$ for the weak component $I_{3}$, hypercharge $Y_{o}$ (or charge $Q=I_{3}+\frac{1}{2} Y_{o}$,), and spinpolarization $\frac{3 i}{2} B \gamma^{1} \gamma^{2}$ operators.

The SM Lagrangian $\mathcal{L}_{F V}$ in Eq. (36) can be equivalently written ${ }^{7}$ in this basis: as derived in Ref. [7], and examined in Ref. [17],

\footnotetext{
${ }^{7}$ The commutator is omitted as the operator acts trivially on one side.
} 


$$
\begin{aligned}
\mathcal{L}_{F V}= & \operatorname{tr}\left\{\boldsymbol { \Psi } _ { q L } ^ { \dagger } ( x ) \left[i \partial_{\mu}+g I^{a} W_{\mu}^{a}(x)\right.\right. \\
& \left.+\frac{1}{2} g^{\prime} Y_{o} B_{\mu}(x)\right] \gamma^{0} \gamma^{\mu} \Psi_{q L}(x) \\
& +\Psi_{t R}^{\dagger}(x)\left[i \partial_{\mu}+\frac{1}{2} g^{\prime} Y_{o} B_{\mu}(x)\right] \gamma^{0} \gamma^{\mu} \Psi_{t R}(x) \\
& \left.+\Psi_{b R}^{\dagger}(x)\left[i \partial_{\mu}+\frac{1}{2} g^{\prime} Y_{o} B_{\mu}(x)\right] \gamma^{0} \gamma^{\mu} \Psi_{b R}(x)\right\} P_{f},
\end{aligned}
$$

while gauge and Lorentz symmetries can be checked with the above transformation rule, or given the equivalence to the traditional formulation. A projection operator $P_{f}$ that connects the two expressions [17] can be omitted by finding phases for $\boldsymbol{\Psi}$, which translates into finding an adequate $\gamma_{\mu}$ basis. The trace coefficient is usually 1 , as the field normalization factor accounts for reducible representations. A complete proof of the equivalence is given in Appendix A.

The $\mathrm{W}$-fermion vertex in $\mathcal{L}_{F V}$, Eq. (38), contains the matrix element $\left\langle F^{\prime}\left|W_{o \mu}^{i}\right| F\right\rangle$, where the $\mathrm{W}$ contribution,

$$
W_{o \mu}^{i}=g \gamma_{0} \gamma_{\mu} I_{i},
$$

describes the $\mathrm{SU}(2)_{L}$ inherently chiral action on fermion states $|F\rangle,\left|F^{\prime}\right\rangle$, as it carries the projection $L_{5}=\frac{1}{2}\left(1-\tilde{\gamma}_{5}\right)$, predicted by the spin basis [9]; it is thus the natural choice. For example, this property is absent for $W_{o \mu}^{\prime i}=g \gamma_{0} \gamma_{\mu} I_{i}^{\prime}$, where $I_{i}^{\prime}$ are the $\mathrm{SU}(2)_{L}$ generators without $L_{5}$. Although an equivalent interaction term results within this space, it requires the inclusion of $L_{5}$ within the vertex; worse, $\left[Y_{o}, I_{1,2}^{\prime}\right] \neq 0$.

\section{SCALAR-VECTOR LAGRANGIAN: EXTENDED CHARGE-CONJUGATE SYMMETRY}

\section{A. Conventional $\mathcal{L}_{S V}$}

In the SM, the Higgs particle is present [1] in the $\mathrm{SU}(2)_{L} \times \mathrm{U}(1)_{Y}$ gauge-invariant interacting Lagrangiandensity component

$$
\mathcal{L}_{S V}=\mathbf{H}^{\dagger}(x) \mathbf{F}^{\mu \dagger}(x) \mathbf{F}_{\mu}(x) \mathbf{H}(x),
$$

with

$$
\mathbf{F}_{\mu}(x)=i \partial_{\mu}+\frac{1}{2} g \boldsymbol{\tau} \cdot \mathbf{W}_{\mu}(x)+\frac{1}{2} g^{\prime} B_{\mu}(x),
$$

$\mathbf{W}_{\mu}(x)=\left(W_{\mu}^{1}(x), W_{\mu}^{2}(x), W_{\mu}^{3}(x)\right)$, and the $Y=1$ complexdoublet scalar

$$
\mathbf{H}(x)=\frac{1}{\sqrt{2}}\left(\begin{array}{l}
\eta_{1}(x)+i \eta_{2}(x) \\
\eta_{3}(x)+i \eta_{4}(x)
\end{array}\right),
$$

composed of two charged (upper), and two neutral (lower) fields.

\section{B. $\mathcal{L}_{S V}$ with Higgs and conjugate}

$\mathcal{L}_{S V}$ can be equivalently written (with $B_{\mu}(x) \rightarrow-B_{\mu}(x)$ ) in terms of the orthogonal $Y=-1$ combination $\tilde{\mathbf{H}}(x)=$ $i \tau_{2} \mathbf{H}^{*}(x)$, which uses an antiunitary transformation $\mathcal{C}$ expressing charge-conjugation invariance (in addition to the $C P$ symmetry in the electroweak sector, and approximate $\mathrm{SU}(2)_{L} \times \mathrm{SU}(2)_{R}$ symmetry [18]; a Hilbert space is assumed;) this is also a consequence of the $\mathrm{SU}(2)$ property that the conjugate representation is obtained from a similarity transformation, which ensures independence of the doublet choice. Appendix B shows that

$$
\mathcal{L}_{S V}=\operatorname{tr}\left[\mathbf{F}^{\prime}{ }_{\mu} \overline{\mathbf{H}}_{\chi_{1} \chi_{b}}(x)\right]^{\dagger} \mathbf{F}^{\prime \mu} \overline{\mathbf{H}}_{\chi_{t} \chi_{b}}(x),
$$

where $\overline{\mathbf{H}}_{\chi_{t} \chi_{b}}(x)=\left(\chi_{t} \mathbf{H}(x), \chi_{b} \tilde{\mathbf{H}}(x)\right)$ is a $4 \times 4$ matrix, $\chi_{t}$, $\chi_{b}$ are complex, and $\left|\chi_{t}\right|^{2}+\left|\chi_{b}\right|^{2}=1$, with

$$
\begin{aligned}
\mathbf{F}^{\prime}{ }_{\mu} \overline{\mathbf{H}}_{\chi_{t} \chi_{b}}(x)= & \left(i \partial_{\mu}+\frac{1}{2} g \boldsymbol{\tau} \cdot \mathbf{W}_{\mu}(x)\right) \overline{\mathbf{H}}_{\chi_{t} \chi_{b}}(x) \\
& +g^{\prime} \overline{\mathbf{H}}_{\chi_{1} \chi_{b}}(x) B_{\mu}(x) \tau_{3},
\end{aligned}
$$

which is diagonal in $\mathbf{H}(x), \tilde{\mathbf{H}}(x)$, and hence does not mix them. Moreover, $\mathcal{L}_{S V}$ is a sum of weighted positive-definite terms, meaning only the combination $\left|\chi_{t}\right|^{2}+\left|\chi_{b}\right|^{2}$ results. This generalizes the expression $[19,20]$ for $\mathcal{L}_{S V}$ in terms of $\overline{\mathbf{H}}_{\frac{1}{\sqrt{2}} \frac{1}{\sqrt{2}}}(x)$. With the U(1) overall phase, a three-parameter subspace of the norm-conserving constraint $\left|\chi_{t}\right|^{2}+\left|\chi_{b}\right|^{2}=$ 1 is generated. We associate this isometry with the $\mathcal{L}_{S V}$ invariance under $\mathcal{C}:-\tau_{2} \mathcal{K} F^{\prime}{ }_{\mu} \overline{\mathbf{H}}_{\chi_{t} \chi_{b}}(x) \tau_{2} \mathcal{K}=F^{\prime}{ }_{\mu} \overline{\mathbf{H}}_{\chi_{b}^{*} \chi_{t}^{*}}(x)$, with $\mathcal{K}$ the complex conjugate operator; $\mathcal{L}_{S V}$ is also invariant under the $\tau_{3}$ transformation defined as $\overline{\mathbf{H}}_{\chi_{1} \chi_{b}}(x) \rightarrow$ $\overline{\mathbf{H}}_{\chi_{1} \chi_{b}}(x) \tau_{3}$, together with the combination $\mathcal{C} \tau_{3}$.

Further extension can be made for the scalars in the spin basis by attaching the $\tilde{\gamma}_{5}$ operator. Using the projection operators in Eq. (14), $\mathcal{L}_{S V}$ in Eq. (42) is generalized with the substitutions

$$
\begin{gathered}
\mathbf{F}_{\mu}^{\prime} \rightarrow\left(L_{5}\right)_{4 \times 4} \mathbf{F}_{\mu}^{\prime} \\
\overline{\mathbf{H}} \rightarrow\left(L_{5}\right)_{4 \times 4}\left(\gamma_{0}\right)_{4 \times 4} \overline{\mathbf{H}},
\end{gathered}
$$

thus including spin d.o.f., leading to a combined spinorelectroweak description. An intermediate expression that connects to the spin basis, and ultimately to Yukawa components, is obtained

$$
\begin{aligned}
\mathcal{L}_{S V}= & \frac{1}{2} \operatorname{tr}\left[L_{5} \mathbf{F}^{\prime}{ }_{\mu} L_{5} \gamma_{0} \overline{\mathbf{H}}_{\chi_{t} \chi_{b}}(x)\right]^{\dagger} L_{5} \mathbf{F}^{\prime \mu} L_{5} \gamma_{0} \overline{\mathbf{H}}_{\chi_{t} \chi_{b}}(x) \\
= & \frac{1}{4} \operatorname{tr}\left(\left[L_{5} \mathbf{F}^{\prime}{ }_{\mu} L_{5} \gamma_{0} \overline{\mathbf{H}}_{\chi_{t} \chi_{b}}(x)\right]^{\dagger}+L_{5} \mathbf{F}^{\prime}{ }_{\mu} L_{5} \gamma_{0} \overline{\mathbf{H}}_{\chi_{t} \chi_{b}}(x)\right) \\
& \left(L_{5} \mathbf{F}^{\prime \mu} L_{5} \gamma_{0} \overline{\mathbf{H}}_{\chi_{1} \chi_{b}}(x)+\left[L_{5} \mathbf{F}^{\prime \mu} L_{5} \gamma_{0} \overline{\mathbf{H}}_{\chi_{\iota} \chi_{b}}(x)\right]^{\dagger}\right),
\end{aligned}
$$


with the trace also over spin d.o.f., the second equality using hermitian conjugates, $R_{5} L_{5}=L_{5} R_{5}=0$, and trace properties which lead to only two identical nontrivial terms. These forms will prove useful in comparing with Yukawa terms below.

\section{C. $\mathcal{L}_{S V}$ in $(7+1)$-dimensional spin space}

In the spin basis, the four-scalar doublet structure above is reproduced. Indeed, it emerges naturally in the $(7+1)$-dimensional spin basis, with the Higgs potential not altered under different definitions (chiral ones or not.) Table I presents two of these scalar elements (with two additional as their conjugates.) Together with coordinate dependence, they are

$$
\begin{aligned}
& \boldsymbol{\phi}_{1}(x)=\frac{1}{\sqrt{2}}\left[\eta_{1}(x)+i \eta_{2}(x)\right] \phi_{1}^{+}+\frac{1}{\sqrt{2}}\left[\eta_{3}(x)+i \eta_{4}(x)\right] \phi_{1}^{0} \\
& \boldsymbol{\phi}_{2}(x)=\frac{1}{\sqrt{2}}\left[\eta_{1}(x)+i \eta_{2}(x)\right] \phi_{2}^{+}+\frac{1}{\sqrt{2}}\left[\eta_{3}(x)+i \eta_{4}(x)\right] \phi_{2}^{0},
\end{aligned}
$$

and whose quantum numbers associate them to the Higgs doublet. These are unique within the $(7+1)$-dimensional space [9]. Although new scalar fields are introduced in principle, here we concentrate on the SM-equivalent projections. Given the SM Higgs conjugate representation $\tilde{\mathbf{H}}(x)$ the scalar components are interpreted through the assignments (see Table I),

$$
\begin{aligned}
\mathbf{H}(\mathbf{x}) & \rightarrow \phi_{1}(x)-\boldsymbol{\phi}_{2}(x) \\
\tilde{\mathbf{H}}^{\dagger}(x) & \rightarrow \phi_{1}(x)+\boldsymbol{\phi}_{2}(x) .
\end{aligned}
$$

This leads to the equivalent expressions,

$$
\begin{aligned}
\mathcal{L}_{S V}= & \operatorname{tr}\left\{\left[\mathbf{F}^{\prime \prime}(x), \mathbf{H}_{a f}(x)\right]_{ \pm}^{\dagger}\left[\mathbf{F}^{\prime \prime}(x), \mathbf{H}_{a f}(x)\right]_{ \pm}\right\}_{\mathrm{sym}} \\
= & \frac{1}{2} \operatorname{tr}\left\{[ \mathbf { F } ^ { \prime \prime } ( x ) , \mathbf { H } _ { a f } ( x ) + \mathbf { H } _ { a f } ^ { \dagger } ( x ) ] _ { \pm } ^ { \dagger } \left[\mathbf{F}^{\prime \prime}(x), \mathbf{H}_{a f}(x)\right.\right. \\
& \left.\left.+\mathbf{H}_{a f}^{\dagger}(x)\right]_{ \pm}\right\}_{\mathrm{sym}},
\end{aligned}
$$

where we introduced $\mathbf{H}_{a f}(x)=a \boldsymbol{\phi}_{1}(x)+f \boldsymbol{\phi}_{2}(x)$, and

$$
\mathbf{F}^{\prime \prime}(x)=\left[i \partial_{\mu}+g W_{\mu}^{i}(x) I_{i}+\frac{1}{2} g^{\prime} B_{\mu}(x) Y_{o}\right] \gamma_{0} \gamma^{\mu}
$$

the subindex sym means only symmetric $\gamma_{\mu} \gamma_{\nu}$ components are taken, to avoid the Pauli components; and the \pm index means the commutator and the anticommutator should be used for the temporal and spatial $\gamma_{\mu}$ components, respectively. The equality for $\mathcal{L}_{S V}$ implies that it accommodates SM parity-conserving scalar representations. The complex parameters $a, f$, are constrained by the normalization rule $|a|^{2}+|f|^{2}=1$. These properties for $\mathcal{L}_{S V}$ are shown explicitly in Appendix B.

\section{D. $\mathcal{L}_{S V}$ mass components in conventional and $(7+1)$-dimensional spin space}

The spin representation can be connected with that of $\overline{\mathbf{H}}_{\chi_{1} \chi_{b}}(x)$ with the expression $\mathbf{H}_{a f}(x)=\frac{1}{\sqrt{2}}\left(\chi_{t} \mathbf{H}_{t}(x)+\right.$ $\left.\chi_{b} \mathbf{H}_{b}(x)\right)$, where

$$
\begin{aligned}
\mathbf{H}_{t}(x) & =\boldsymbol{\phi}_{1}(x)+\boldsymbol{\phi}_{2}(x) \\
\mathbf{H}_{b}(x) & =\boldsymbol{\phi}_{1}(x)-\boldsymbol{\phi}_{2}(x),
\end{aligned}
$$

with $\boldsymbol{\phi}_{i}$ defined in Eq. (48), and this parametrization applies the unitary transformation $\chi_{t}=\frac{1}{\sqrt{2}}(a+f), \chi_{b}=\frac{1}{\sqrt{2}}(a-f)$.

Under the Higgs mechanism, the SM scalars acquire $[2,3]$ a vacuum expectation value $v$, and only the neutral field $\eta_{3}(x)$ survives: $\left\langle\eta_{3}(x)\right\rangle=v,\langle\mathbf{H}(x)\rangle=\frac{v}{\sqrt{2}}\left(\begin{array}{l}0 \\ 1\end{array}\right)$, while the charged and imaginary components are absorbed into vector bosons, as seen explicitly in the unitary gauge. Idem in the spin basis, as can be proved by the Lagrangian equivalence or directly; then,

$$
\left\langle\mathbf{H}_{a f}(x)\right\rangle=H_{n}=\frac{v}{2}\left(\chi_{t} H_{t}^{0}+\chi_{b} H_{b}^{0}\right),
$$

where the normalized Higgs operator $H_{n}$ is defined, with the same $0,+$ component conventions as for the $\phi_{i}$, implying, as $\operatorname{tr} H_{i}^{0 \dagger} H^{0}{ }_{j}=2 \delta_{i j}, i, j=t, b$,

$$
\begin{aligned}
\left\langle\mathbf{H}_{a f}^{\dagger}(x) \mathbf{H}_{a f}(x)\right\rangle & =\left(|a|^{2}+|f|^{2}\right) v^{2} / 2 \\
& =\left(\left|\chi_{t}\right|^{2}+\left|\chi_{b}\right|^{2}\right) v^{2} / 2=v^{2} / 2 .
\end{aligned}
$$

The vector-Higgs vertex in $\mathcal{L}_{S V}$ determines the vectorboson masses, and within the spin basis, the trace is taken consistently with $H_{n}$. Thus, the mass component, extracted from Eq. (50), taking for $\mathbf{F}^{\prime \prime}$ the W, Z field terms, and for $\mathbf{H}_{a f}$ its vacuum expectation value in Eq. (54),

$$
\begin{aligned}
\mathcal{L}_{S V m}= & \operatorname{tr}\left(\left[H_{n}, g W_{0}^{m}(x) I_{m}+\frac{g^{\prime}}{2} Y_{o} B_{0}(x)\right]^{\dagger}\right. \\
& {\left[H_{n}, g W_{0}^{l}(x) I_{l}+\frac{g^{\prime}}{2} Y_{o} B_{0}(x)\right] } \\
& +\left\{H_{n},\left(g W_{i}^{k}(x) I_{k}+\frac{g^{\prime}}{2} Y_{o} B_{i}(x)\right) \gamma_{0} \gamma^{i}\right\}^{\dagger} \\
& \left.\left\{H_{n},\left(g W_{j}^{l}(x) I_{l}+\frac{g^{\prime}}{2} Y_{o} B_{j}(x)\right) \gamma_{0} \gamma^{j}\right\}\right)
\end{aligned}
$$

is produced. For the neutral massive vector boson, one derives the normalized $Z_{\mu}(x)=\left(-g W_{\mu}^{3}(x)+g^{\prime} B_{\mu}(x)\right) /$ $\sqrt{g^{2}+g^{\prime 2}}$, and massless photon $A_{\mu}(x)=\left(g^{\prime} W_{\mu}^{3}(x)+\right.$ $\left.g B_{\mu}(x)\right) / \sqrt{g^{2}+g^{\prime 2}}$, giving, e.g., the 0-component 


$$
\begin{aligned}
\mathcal{L}_{S Z m 0}= & \operatorname{tr}\left[H_{n}, W_{0}^{3}(x) g I_{3}+B_{0}(x) \frac{1}{2} g^{\prime} Y_{o}\right]^{\dagger} \\
& {\left[H_{n}, W_{0}^{3}(x) g I_{3}+B_{0}(x) \frac{1}{2} g^{\prime} Y_{o}\right] } \\
= & Z_{0}^{2}(x) \frac{1}{g^{2}+g^{\prime 2}} \operatorname{tr}\left[H_{n}, g^{2} I_{3}-\frac{1}{2} g^{\prime 2} Y_{o}\right]^{\dagger} \\
& {\left[H_{n}, g^{2} I_{3}-\frac{1}{2} g^{\prime 2} Y_{o}\right]=\frac{1}{2} Z_{0}^{2}(x) m_{Z}^{2}, }
\end{aligned}
$$

implying

$$
\begin{aligned}
& \operatorname{tr} \frac{1}{g^{2}+g^{\prime 2}}\left[\sqrt{2} H_{n}, g^{2} I_{3}-\frac{1}{2} g^{\prime 2} Y_{o}\right]^{\dagger}\left[\sqrt{2} H_{n}, g^{2} I_{3}-\frac{1}{2} g^{\prime 2} Y_{o}\right] \\
& \quad=v^{2}\left(g^{2}+g^{\prime 2}\right) / 4
\end{aligned}
$$

thus, $m_{Z}=v \sqrt{g^{2}+g^{\prime 2}} / 2, m_{A}=0$.

Similarly, for $\mathcal{L}_{S W m}$, the $W_{o \mu}^{i}$ basis in Eq. (39) emerges, and defines the masses of the charged boson fields $W_{\mu}^{ \pm}(x)=\frac{1}{\sqrt{2}}\left(W_{\mu}^{1}(x) \mp i W_{\mu}^{2}(x)\right)$. Thus, the charged-vector boson component,

$$
\begin{aligned}
\mathcal{L}_{S W m 0} & =W_{0}^{i}(x) W_{0}^{j}(x) \operatorname{tr}\left[H_{n}, W_{o 0}^{i}\right]^{\dagger}\left[H_{n}, W_{o 0}^{j}\right] \\
& =m_{W}^{2} W_{0}^{+}(x) W_{0}^{-}(x),
\end{aligned}
$$

$i, j=1,2$, contains $m_{W}^{2}=\operatorname{tr}\left[H_{n}, W_{o 0}^{+}\right]^{\dagger}\left[H_{n}, W_{o 0}^{+}\right]=v^{2} g^{2} / 4$, with $W_{o \mu}^{ \pm}=\frac{1}{\sqrt{2}} g \gamma_{0} \gamma_{\mu} I^{ \pm}, I^{ \pm}=I_{1} \pm i I_{2}$. This assignment is unique as this is the only way to maintain not only the vertex condition (gauge invariance,) but also normalization (above.) When written in terms of $H=H_{n}+H_{n}^{\dagger}$, interpreted as a fermion Hamiltonian, $m_{W}^{2}=\operatorname{tr}\left[H, W_{o 0}^{+}\right]^{\dagger}\left[H, W_{o 0}^{+}\right]$ and the other part is not affected, as $\left[H_{n}^{\dagger}, W_{o \mu}^{+}\right]=0$.

\section{SCALAR-FERMION LAGRANGIAN: HEAVY-QUARK DOUBLET'S MASS CONSTRAINT}

The Yukawa fermion-scalar interaction can be similarly parametrized in the Clifford basis,

$$
\begin{aligned}
-\mathcal{L}_{S F}= & \operatorname{tr} \frac{\sqrt{2}}{v}\left[m_{t} \Psi_{t R}^{\dagger}(x) \mathbf{H}_{t}(x) \boldsymbol{\Psi}_{q L}(x)\right. \\
& \left.+m_{b} \Psi_{q L}^{\dagger}(x) \mathbf{H}_{b}(x) \Psi_{b R}(x)\right]+\{\text { H.c. }\},
\end{aligned}
$$

where $m_{t}$ and $m_{b}$ are the top and bottom masses, respectively, and the fermion fields $\Psi$ are defined in Eq. (37). We note that the Higgs scalar components have the correct chiral action over fermions: under the projection operators in Eq. (14) $L_{5}$, and $R_{5}$, e.g., $R_{5} \mathbf{H}_{t}(x) L_{5}=\mathbf{H}_{t}(x)$, $L_{5} \mathbf{H}_{b}(x) R_{5}=\mathbf{H}_{b}(x), \quad L_{5} \mathbf{H}_{t}(x) R_{5}=0, \quad R_{5} \mathbf{H}_{b}(x) L_{5}=0$. For Eq. (60), the underlying mass operator is $\mathbf{H}_{m}(x)=$ $\frac{\sqrt{2}}{v}\left(m_{t} \mathbf{H}_{t}(x)+m_{b} \mathbf{H}_{b}(x)\right)$, giving, under the Higgs mechanism,
TABLE IV. (a) Quantum numbers of massless left-handed quark weak isospin doublet, and (b) right-handed singlets, with momentum along $\pm \hat{\mathbf{z}}$, given explicitly in Tables II and III. The spin component along $\hat{\mathbf{z}}, \frac{3 i}{2} B \gamma^{1} \gamma^{2}$, is used.

(a) Hypercharge 1/3 left-handed

\begin{tabular}{lccc} 
doublet & $I_{3}$ & $Q$ & $\frac{3 i}{2} B \gamma^{1} \gamma^{2}$ \\
\hline$\left(\begin{array}{c}T_{L}^{1} \\
B_{L}^{1}\end{array}\right)=\left(\begin{array}{c}U_{1 L}^{1} \\
D_{1 L}^{1}\end{array}\right)$ & $1 / 2$ & $2 / 3$ & $1 / 2$ \\
& $-1 / 2$ & $-1 / 3$ & $1 / 2$
\end{tabular}

\begin{tabular}{lccc}
\hline \hline (b) $I_{3}=0$ right-handed singlets & $Y$ & $Q$ & $\frac{3 i}{2} B \gamma^{1} \gamma^{2}$ \\
\hline$T_{R}^{1}=U_{1 R}^{1}$ & $4 / 3$ & $2 / 3$ & $1 / 2$ \\
$B_{R}^{1}=D_{1 R}^{1}$ & $-2 / 3$ & $-1 / 3$ & $1 / 2$ \\
\hline \hline
\end{tabular}

$$
\left\langle\mathbf{H}_{m}(x)\right\rangle=H_{m}=m_{t} H_{t}^{0}+m_{b} H_{b}^{0} .
$$

Examples of quark massive basis states are summarized on Table V (see Tables II-IV), for both u- and d-type quarks, with their quantum numbers. Only one polarization and one flavor are shown, as a more thorough treatment of the fermion-flavor states is given elsewhere [9].

This results in, e.g.,

$$
\begin{array}{ll}
H_{m}^{h} T_{M}^{1}=m_{t} T_{M}^{1}, & H_{m}^{h} T_{M}^{c 1}=-m_{t} T_{M}^{c 1}, \\
H_{m}^{h} B_{M}^{1}=m_{b} B_{M}^{1}, & H_{m}^{h} B_{M}^{c 1}=-m_{b} B_{M}^{c 1},
\end{array}
$$

where $H_{m}^{h}=H_{m}+H_{m}^{\dagger}$, and $T_{M}^{c 1}, B_{M}^{c 1}$ correspond to negative-energy solution states (and similarly for opposite spin components) and Eq. (62) justifies the $m_{t}$ and $m_{b}$ mass interpretation.

Under the assumption of a single mass-producing field operator, we match a reparametrized $H_{n}$ in Eq. (58) that gives the $\mathrm{Z}$ mass, to the fermion-mass term $H_{m}$, in Eq. (62), resulting in $\sqrt{2} H_{n}=H_{m}$; a multiplet structure is suggested. In other words, the operator identification derives from their mass eigenvalues, expressed schematically as $\left|\left\langle Z\left|\sqrt{2} H_{n}\right| Z\right\rangle\right|^{2}=m_{Z}^{2}$ and $\left\langle t\left|H_{m}+H_{m}^{\dagger}\right| t\right\rangle=m_{t}$, and the proportionality constant is derived accordingly. In this association, the simple real-field $Z_{\mu}(x)$ nature justifies its use [similarly for each $W_{\mu}^{i}(x)$ ], as opposed to the complex $W_{\mu}^{ \pm}(x)$. Similarly, Eq. (50) is chosen over Eq. (51), as the latter adds the Higgs conjugate representation,

TABLE V. Massive quark eigenstates of $H_{m}^{h}$ given after Eq. (62).

\begin{tabular}{lccc}
\hline \hline Massive quarks & $H_{m}^{h}$ & $Q$ & $\frac{3 i}{2} B \gamma^{1} \gamma^{2}$ \\
\hline$T_{M}^{1}=\frac{1}{\sqrt{2}}\left(T_{L}^{1}+T_{R}^{1}\right)$ & $m_{t}$ & $2 / 3$ & $1 / 2$ \\
$B_{M}^{1}=\frac{1}{\sqrt{2}}\left(B_{L}^{1}-B_{R}^{1}\right)$ & $m_{b}$ & $-1 / 3$ & $1 / 2$ \\
$T_{M}^{c 1}=\frac{1}{\sqrt{2}}\left(T_{L}^{1}-T_{R}^{1}\right)$ & $-m_{t}$ & $2 / 3$ & $1 / 2$ \\
$B_{M}^{c 1}=\frac{1}{\sqrt{2}}\left(B_{L}^{1}+B_{R}^{1}\right)$ & $-m_{b}$ & $-1 / 3$ & $1 / 2$ \\
\hline \hline
\end{tabular}


unlike the SM. Thus, the vacuum expectation value reproduces the parametrization in Eq. (54) and identifies $\chi_{t}, \chi_{b}$ as Yukawa parameters:

$$
\chi_{t}=m_{t} / \frac{v}{\sqrt{2}}, \quad \chi_{b}=m_{b} / \frac{v}{\sqrt{2}}
$$

The same argument can be made using the second scalar form in Eq. (51), as it also leads to Eq. (58). This results in

$$
\begin{aligned}
-\mathcal{L}_{S F}= & \operatorname{tr} \sqrt{2}\left[\Psi_{t R}^{\dagger}(x) \mathbf{H}_{a f}(x) \boldsymbol{\Psi}_{q L}(x)\right. \\
& \left.+\boldsymbol{\Psi}_{q L}^{\dagger}(x) \mathbf{H}_{a f}(x) \Psi_{b R}(x)\right]+\{\text { H.c. }\}
\end{aligned}
$$

Using Eq. (55), we obtain the relation for the $t, b$ quark masses:

$$
\left(|a|^{2}+|f|^{2}\right) v^{2} / 2=\left|m_{t}\right|^{2}+\left|m_{b}\right|^{2}=v^{2} / 2 .
$$

The commutator arrangement in Eq. (57) is used in the above comparison; as it is set on the demand of a normalized scalar, the argument strengthens on the use of the same $\mathrm{Z}$ operator acting on fermions in Eq. (38). The coefficient matching in $\mathcal{L}_{S F}$ derives from the underlying freedom of choice in $\mathcal{L}_{S V}$, and, in turn, from the underlying three-parameter $\tau_{3}-\mathcal{C}$ symmetry that can be equally implemented in the spin basis. Looking at the matrix structure, the $\gamma_{0}$ operator within $H_{m}$ makes it a rank-2 reduciblerepresentation operator, as expressed in Eq. (54) and can be read in Eq. (46); indeed, $H_{m}$ connects two fermion spin polarizations, but hits a single $\mathrm{W}$ state's components twice as $H_{m}$ duplicates the scalar representations, requiring the $\frac{1}{\sqrt{2}}$ normalization factor. In yet another interpretation, this relation is obtained from the normalization restriction in the Yukawa term in Eq. (55), dividing out the energy scale set by the vacuum expectation. To the extent that these arguments rely on a common metric vector space, they are geometric.

Equation (65) assumes the parity-conserving condition, constraining the quark masses. ${ }^{8}$ For maximal hierarchy [7], with $a, f$ dependence on one comparable large scale $\mathrm{O}(a) \simeq \mathrm{O}(f),\left(m_{b} \ll m_{t}\right)$, we get $\frac{1}{\sqrt{2}} v \simeq 173.95$, for $v=246 \mathrm{GeV}, m_{b}=0$; alternatively, the quark-b mass input predicts the top quark-mass as $m_{t}=\sqrt{v^{2} / 2-m_{b}^{2}} \simeq 173.90 \mathrm{GeV}$, for [4] $m_{b}=4 \mathrm{GeV}$ (while renormalization effects give [21] $m_{b}\left(m_{t}\right) \sim$ $2 \mathrm{GeV}$.) These two calculations are consistent with the measured top pole mass [4] $\bar{m}_{t}=173.21 \pm 0.51 \pm$ $0.71 \mathrm{GeV}$, where systematic and statistic errors are quoted, respectively. Future precision improvements will

\footnotetext{
${ }^{8}$ We neglect t-b mixing as the CKM matrix is nearly diagonal [4], confirming this method can be applied here.
}

test the limits of this tree-level calculation, with view of the bottom-quark influence.

\section{EXTENDED QUARK-MASS RELATION}

We place the heavy-quark mass relation in Eq. (65) in the larger SM context, and argue for a plausible generalization for all quarks, based on it. For these purposes, we first derive some SM field properties using the spin basis, assuming they can be also derived within the conventional SM basis, given their equivalent application. Needless to say, we demand consistency with the SM, and with experiment. At the section's end, we identify some underlaying general assumptions.

Thus, we concentrate on the SM three-generation subset of the $(7+1)$-dimensional model [9], as can be effected by the Yukawa operators in Eq. (34). Equation (65) uses that the same single-scalar operator acts on the fermions and the vector bosons: such an operator is reproduced in the $S V$ and $S F$ terms, as the $S V$ term admits a basis expression that applies the associated $\mathcal{C}$-symmetries in Sec. IV. This connection implies the equivalent expression that can be read from the Appendixes,

$$
\mathcal{L}_{S V}=\left|\chi_{t}\right|^{2} \mathcal{L}_{S V u}+\left|\chi_{b}\right|^{2} \mathcal{L}_{S V d},
$$

which shows separation of quark $i=\mathrm{u}$ - and d-type $\mathcal{L}_{S V i}$ components, depending on scalars, and no mixing among them. We focus on the mass-generating scalar elements corresponding to the neutral $H_{t}^{0}, H_{b}^{0}$, from Eqs. (53) and (54), and their hermitian conjugates. As mass relations are considered, we assume fields after the Higgs mechanism is applied.

In particular, a connection emerges between the normalized bilinear Higgs term that gives masses to the vector bosons, as the $\mathrm{Z}$ mass in Eq. (58), and the fermions.

$$
\begin{aligned}
\frac{1}{2} \operatorname{tr} H_{m}^{\dagger} H_{m}= & 2 \operatorname{tr}\left[\left(H_{m} T_{L}^{\alpha} T_{R}^{\alpha \dagger}\right)^{\dagger} H_{m} T_{L}^{\alpha} T_{R}^{\alpha \dagger}\right. \\
& \left.+\left(H_{m} B_{R}^{\alpha} B_{L}^{\alpha \dagger}\right)^{\dagger} H_{m} B_{R}^{\alpha} B_{L}^{\alpha \dagger}\right] \\
= & 2 \operatorname{tr}\left[H_{m}^{\dagger} H_{m} T_{L}^{\alpha} T_{R}^{\alpha \dagger} T_{R}^{\alpha} T_{L}^{\alpha \dagger}\right. \\
& \left.+H_{m}^{\dagger} H_{m} B_{R}^{\alpha} B_{L}^{\alpha \dagger} B_{L}^{\alpha} B_{R}^{\alpha \dagger}\right] \\
= & v^{2}\left(\chi_{t}^{2}+\chi_{b}^{2}\right),
\end{aligned}
$$

where $H_{m}$ is defined in Eq. (61), $T_{R}^{\alpha}, T_{L}^{\alpha}, B_{R}^{\alpha}, B_{L}^{\alpha}$, are quarks at rest, defined in Table IV, $\chi_{t}, \chi_{b}$ are $H_{t}^{0}, H_{b}^{0}$ coefficients, as given in Eq. (54), in the second equality we use the trace property, and the third expresses the $H_{m}$ normalization condition. Factor 2 comes as only one spin fermion component is used. Thus, $\mathcal{L}_{S V}$ elements can be written as a sum of inner products between Yukawa and SM scalar components. This relation derives from the projective nature of Higgs normal and dual terms, accompanied by a fermion chirality operator in $\mathbf{H}_{t}(x), \mathbf{H}_{b}(x)$ in Eq. (53). 
Equation (67) can also be understood from the substitutions in $\operatorname{tr} H_{m}^{\dagger} H_{m}$

$$
\begin{aligned}
& H_{t}^{0} \rightarrow H_{t}^{0} T_{L}^{\alpha} T_{R}^{\alpha \dagger}=\frac{1}{\chi_{t}} y_{q t}^{U} H_{t}^{0} T_{L}^{\alpha} Y_{q t}^{F} T_{R}^{\alpha \dagger} \\
& H_{b}^{0} \rightarrow H_{b}^{0} B_{R}^{\alpha} B_{L}^{\alpha \dagger}=\frac{1}{\chi_{b}} y_{b q}^{D} H_{b}^{0} B_{R}^{\alpha} Y_{b q}^{F} B_{L}^{\alpha \dagger},
\end{aligned}
$$

with terms extracted from $\mathcal{L}_{S F}$ in Eq. (60), using the trace permutation property. The identity in each substitution provides the link to the $t, b$ Yukawa constants for the $q=t b$ doublet, $t, b$ singlet cases. The arguments leading to the mass relation in Eq. (65) imply $y_{q t}^{U}=\chi_{t}, y_{b q}^{D}=\chi_{b}$, as given in Eq. (63), namely, a diagonal mass basis is assumed.

Since one can pick any fermion generation on Tables II and III, the interpretation of the $\chi_{t}, \chi_{b}$ coefficients as Yukawa constants within the $S V$ term leads to a generalization to other families and nondiagonal Yukawa elements. We now consider the extension of $\mathcal{L}_{S F}$ in Eqs. (60) and (64) with a fermion expansion that uses all Yukawa coefficients,

$$
\begin{aligned}
-\mathcal{L}_{S F T}= & \operatorname{tr}\left[\sum_{i q} y_{i q}^{U} \boldsymbol{\Psi}_{i R}^{\dagger}(x) \mathbf{H}_{t}(x) \boldsymbol{\Psi}_{q L}(x) Y_{q i}^{F}\right. \\
& \left.+\sum_{j q} y_{q j}^{D} \boldsymbol{\Psi}_{q L}^{\dagger}(x) \mathbf{H}_{b}(x) \Psi_{j R}(x) Y_{j q}^{F}\right]+\{\text { H.c. }\},
\end{aligned}
$$

where the Yukawa operators $Y^{F}$ from Eq. (34) are necessary to connect the u- and d-type quark fields defined in Eq. (37), and $y_{q i}^{U}, y_{q j}^{D}$ are Yukawa coefficients, with the up, down, charm and strange quarks, also included, relabelling singlets $i=u, c, t, j=d, s, b$, and doublets $q=u d, c s, t b$.

The allowed Yukawa terms, diagonal and mixed, can be included using all combinations of a three-generation set of normalized fermions on Table II, where a projection operator as in Eq. (35) is applied. We evaluate the trace of bilinear $F_{U}=\frac{1}{\chi_{t}} y_{q i}^{U} U_{q L}^{\alpha} Y_{q i}^{F} U_{i R}^{\alpha}{ }^{\dagger}, F_{D}=\frac{1}{\chi_{b}} y_{j q^{\prime}}^{D} D_{j R}^{\alpha} Y_{j q^{\prime}}^{F} D_{q^{\prime} L}^{\alpha}{ }^{\dagger}$ terms with $\mathcal{L}_{S V}$ components, extending Eq. (67), producing

$$
\begin{aligned}
2 \operatorname{tr} & {\left[\left(H_{m} F_{U}\right)^{\dagger} H_{m} F_{U}+\left(H_{m} F_{D}\right)^{\dagger}\left(H_{m} F_{D}\right)\right] } \\
& =\frac{1}{2} v^{2}\left(\left|y_{q i}^{U}\right|^{2} \operatorname{tr} H_{t}^{0 \dagger} H_{t}^{0}+\left|y_{j q^{\prime}}^{D}\right|^{2} \operatorname{tr} H_{b}^{0 \dagger} H_{b}^{0}\right) \\
& =v^{2}\left(\left|y_{q i}^{U}\right|^{2}+\left|y_{j q^{\prime}}^{D}\right|^{2}\right),
\end{aligned}
$$

which may be also obtained by the substitution of the associated scalar coefficients in bilinear neutral Higgs terms $\operatorname{tr} H_{m}^{\dagger} H_{m}$,

$$
\begin{aligned}
& H_{t}(x) \rightarrow H_{t}^{0} F_{U}=H_{t}^{0} \frac{1}{\chi_{t}} y_{q i}^{U} U_{q L}^{\alpha} Y_{q i}^{F} U_{i R}^{\alpha}{ }^{\dagger} \\
& H_{b}(x) \rightarrow H_{b}^{0} F_{D}=H_{b}^{0} \frac{1}{\chi_{b}} y_{j q^{\prime}}^{D} D_{j R}^{\alpha} Y_{j q^{\prime}}^{F} D_{q^{\prime} L}^{\alpha}{ }^{\dagger}
\end{aligned}
$$

The correspondence of $\mathcal{L}_{S F}$ in Eqs. (60) and (64) to $\mathcal{L}_{S F T}$ in Eq. (69) induces the sum of square mass-matrix elements in Eq. (70), which is equal (given the property $\operatorname{tr} M^{\dagger} M=$ $\operatorname{tr} M^{\prime \dagger} M^{\prime}, M$ a matrix, $M^{\prime}$ its diagonal form) to the sum over the square masses,

$$
v^{2}\left(\sum_{q i}\left|y_{q i}^{U}\right|^{2}+\sum_{q j}\left|y_{q j}^{D}\right|^{2}\right)=2\left(\sum_{i} m_{i}^{2}+\sum_{j} m_{j}^{2}\right) .
$$

A generalization with such a sum is induced, similar to relation Eq. (65), with the Higgs normalization condition, Eq. (55). Since Eq. (70) maintains the same structure as Eq. (67), following the generalization of $\mathcal{L}_{S F}$ to $\mathcal{L}_{S F T}$,

$$
m_{t}^{2}+m_{c}^{2}+m_{u}^{2}+m_{b}^{2}+m_{s}^{2}+m_{d}^{2}=v^{2} / 2 .
$$

Implicitly, we used the $S V$-fermion symmetry, namely, no fermion preference. With today's uncertainties in the quarkmass values, this relation is phenomenologically consistent with Eq. (65), as the same maximal hierarchy or quark b-mass input argument follows, and the rest of the quarks have comparably negligible masses. As this relation is independent of the mass diagonalization matrix, it is also of the CKM matrix [22].

The two quark-mass conditions in Eqs. (65) and (73) are interpreted. This paper shows SM features support a boson and fermion connection leading to the $t, b$ quark mass condition in Eq. (65). If only such quarks belong in the same class as the other massive SM bosons, a different mass-generating mechanism is expected for the other fermions; one concludes that they are not affected by such dynamics, as their masses are comparably negligible. On the other hand, if there is a common dynamics, as suggested by the similar fermion-boson inner product, the all-quark condition Eq. (73) applies, given the fermion symmetry, and the structure similarity between Eqs. (67) and (70).

Initial fermion states within the three-generation set for $\mathcal{L}_{S F T}$ in Eq. (69) remain within such a subspace, given the commuting property of the projection operator $Y^{F 4}$ in Eq. (35) with baryon-number, Lorentz, gauge and mass operators $\left(B, B \sigma_{\mu \nu} I_{i}, Y_{o}, \boldsymbol{\phi}_{i}, i=1,2\right.$.) In other words, within the three-generation states, the substitution $Y^{F 4 \dagger} Y_{i j}^{F} Y^{F 4}$ in $\mathcal{L}_{S F T}$ is valid. This implies that no operator will connect the initial fermions outside the three generations. So is the case for the three-generation extension of $\mathcal{L}_{F V}$ in Eq. (38), requiring a sum over the (electroweak) flavors. We conclude the three-generation spin-basis projection consistently describes the SM.

By construction, Eqs. (68) and (71) imply masses represent $\mathrm{O}\left(m_{q} / m_{t}\right)$ corrections. This is also the order of the Hamiltonian needed to obtain the other fermion masses. More assumptions are necessary to get further information on masses, and CKM matrix elements. For example, hierarchy arguments on the masses' order of 
magnitude difference were derived [9] that explain how the associated $\mathrm{W}, \mathrm{Z}, t, b$, large scale mostly cancels for the other fermions at the vertical level (within a doublet) and horizontal level (between families). This leads to a consistent description in which such mechanisms coexist with the Higgs-generated one. While we produce above further consistency arguments for their parameters, more stringent constraints from the $(7+1)$-dimensions will be tested elsewhere. Other arguments leading to hierarchy exist as textures [23].

We conclude Yukawa coefficients, contained in rest fermions as a device, connect to bilinear scalar combinations containing mass-generating Higgs terms in $\mathcal{L}_{S V}$, keeping the Lorentz or gauge structure of $S V$ unmodified, and ultimately consistently with the SM. We show above $\mathcal{L}_{S F T}$ in Eq. (69) induces a generalized sum rule for the square quark masses in Eq. (73). The latter is a plausible extension of Eq. (65), based on a subset of $\mathcal{L}_{S V}$ terms, after the Higgs mechanism. The same type of argument can be made for leptons, but given their smaller masses, their influence will be lesser, while similar conditions as in Eq. (67) will also lead to PNRS matrix [24] independence.

\section{CONCLUSIONS AND OUTLOOK}

In summary, the formalism used places fields on a basis that simultaneously contains SM bosons and fermions. $S V$ and $S F$ terms are linked through the mass rendering of the scalar operator within them, using the electroweak $S V$ vertex independence of its components acting on different fermion-doublet elements, implicitly expected, but which we now expose. Supporting a SM prediction of a unique scalar, input from the normalized scalar-vector vertex, and the mass-parameter interpretation in the $S F$ vertex, relates $v$ and $m_{t}$, cf Eq. (65), the main result in the paper. The same relation can be argued by considering the scalar operator's matrix rank, or assuming normalized Yukawa components. Based on chiral properties, the same Higgs-operator rule, and a correspondence between fermion-boson inner products and Yukawa terms, a plausible extended sum rule for the fermion square masses is proposed, given in Eq. (73). Both relations are consistent with the SM, given today's particle-mass uncertainties. We conclude the spin basis is a useful platform to obtain, within the SM, the quark-mass electroweak relations.

The central argument input can be also read when $V$ terms in $\mathcal{L}_{F V}$, attached with the projector $L_{5}$ in Eq. (14), are carried into the intermediate $\mathcal{L}_{S V}$ chiral version in Eq. (46) and, after the $1 / 2$ factor cancellation in its mass component, relate to $F$ terms in the Yukawa $\mathcal{L}_{S F}$. The spin-basis gives it further support as it classifies discrete degrees and produces SM features. Thus, the matrix space restricts representations, in turn, exhausting the space; electroweak $V$ fields belong to the adjoint, and $S, F$ fields to the fundamental representations. Additionally, the chiral property in the $F V$ electroweak term, associated to $V$, translates naturally to the $S V$ interaction components. Normalized fields define the Lagrangian terms, setting the trace coefficient, and the stage for the $\mathcal{L}_{S V}, \mathcal{L}_{S F}$ comparison. In the spin-basis context, the $S$ field's chiral property is nominal, but consistent, as $\mathcal{L}_{S V}$ contains the $L_{5}$ projector from $V$, and within $\mathcal{L}_{S F}, S$ acts on chiral fermion components.

The scalar operator acting on vectors and fermions links their matrix elements, connecting parameters. The particles' simultaneous participation in mass generation through the Higgs mechanism and related SM vertices, with assigned representations, implies a description with common dynamics, and at a given energy scale, already at the classical level, and suggests fields belong in a multiplet, supporting a common-origin unification assumption [7].

It follows that the arguments provide a geometric approach to address problems as the electroweak-symmetry breaking origin. The formalism facilitates the fields' composite description, as boson d.o.f. may be written in terms of two fermions'. Expansions in such fields may be useful, independently of whether compositeness is physical or only a device.

Naturalness is hinted at in the $\boldsymbol{\phi}_{1}, \boldsymbol{\phi}_{2}$ associated single scale, which produces a hierarchy effect [7]. Thus, while this symmetry-breaking effect applies for heavy-quark masses, it could be valid also horizontally between generations in accordance with the fermions' low masses. While here we considered the top-quark mass, the other fermions, besides the b-quark, may be included in this scheme, namely, considering bilinear fermion components for scalar particles, but they will have little influence on this result, as their $S F$ interaction is proportional to their masses.

As the spin basis connects the vector and quark sectors, constraints may be derived for SM extensions as supersymmetry [25], composite models that require dynamical symmetry breaking [14] as technicolor [26] or, in an extension of such models, top and bottom quarks [27] that conform condensate-producing massive particles.

Besides the fields' spin representation connecting the scalar operator in two vertices, it highlights chiral components of particles and interactions that maintain their SM equivalence. Indeed, we showed two such valid chiral and nonchiral scalar bases for the $S V$ Lagrangian. This freedom could be clarified in other vertices, as with a SM extension with additional scalar d.o.f., whereas in this paper, we considered only their SM projection.

\section{ACKNOWLEDGMENTS}

The authors acknowledge support from DGAPA-UNAM through Project No. IN112916, and discussions with A. Ayala. 


\section{APPENDIX A: FERMION-VECTOR $\mathcal{L}_{F V}$ FERMION-SCALAR $-\mathcal{L}_{S V}$ LAGRANGIANS}

In this appendix we show the Lagrangians' equivalence in the conventional and spin bases by considering explicit expressions with accompanying wave functions (or fields). With hindsight, we use the same Lagrangian label in both bases.

First, we use an iterative procedure [16] to obtain a $(7+1)$-dimensional $\gamma^{\mu}$ representation. Starting with the Pauli matrices $\sigma^{1}, \sigma^{2}$, and $\sigma^{3}$, we get the $(3+1)$-dimensional representation

$$
\begin{array}{ll}
\alpha^{0}=\sigma^{1} \otimes \sigma^{3} & \alpha^{1}=-i \sigma^{2} \otimes \sigma^{3}, \\
\alpha^{2}=I_{2} \otimes i \sigma^{1} & \alpha^{3}=I_{2} \otimes i \sigma^{2}
\end{array}
$$

then, the $(5+1)$-dimensional representation

$$
\begin{array}{ll}
\beta^{0}=\alpha^{0} \otimes \sigma^{3} & \beta^{1}=\alpha^{1} \otimes \sigma^{3}, \\
\beta^{2}=\alpha^{2} \otimes \sigma^{3} & \beta^{3}=\alpha^{3} \otimes \sigma^{3}, \\
\beta^{5}=I_{4} \otimes i \sigma^{1} & \beta^{6}=I_{4} \otimes i \sigma^{2},
\end{array}
$$

and finally, the $(7+1)$-dimensional representation

$$
\begin{array}{ll}
\gamma^{0}=\beta^{0} \otimes \sigma^{3} & \gamma^{1}=\beta^{1} \otimes \sigma^{3}, \\
\gamma^{2}=\beta^{2} \otimes \sigma^{3} & \gamma^{3}=\beta^{3} \otimes \sigma^{3}, \\
\gamma^{5}=\beta^{5} \otimes \sigma^{3} & \gamma^{6}=\beta^{6} \otimes \sigma^{3}, \\
\gamma^{7}=I_{8} \otimes i \sigma^{1} & \gamma^{8}=I_{8} \otimes i \sigma^{2} .
\end{array}
$$

The commuting property of the Lorentz and scalar symmetry operators implies that they can be represented as a tensor product. To compare with the spin-space basis, we write the conventional-basis generators as tensor products, choosing the $(7+1)$-dimensional space to represent them; thus the spin- $1 / 2$ and $\mathrm{SU}(2)_{L}$ terms, expressed by the $4 \times 4$ Clifford basis, and Pauli matrices, respectively, generalize to, e.g., $\tau_{3} \otimes 1_{s 2 \times 2} \sim I_{3}$ and $1_{w 2 \times 2} \otimes\left[\frac{i}{2} P_{L} \gamma_{1} \gamma_{2}\right]_{2 \times 2} \sim \frac{i}{2} P_{L} \gamma_{1} \gamma_{2}$, with $\tau_{3}$ the 3-Pauli matrix, and corresponding spin and weak isospin unit operators $1_{s 2 \times 2}, 1_{w 2 \times 2}$, respectively.

Similarly, states in the conventional basis can be obtained that are represented in $(7+1)$-dimensional space. For example, a left-handed (L), spin-1/2 polarization (1), top $(\mathrm{T})$, state $|L 1 T\rangle$ satisfies $\frac{1}{2}\left(1-\tilde{\gamma}_{5}\right)|L 1 T\rangle=-|L 1 T\rangle$, $i \frac{1}{2} P_{L} \gamma_{1} \gamma_{2}|L 1 T\rangle=\frac{1}{2}|L 1 T\rangle, I_{3}|L 1 T\rangle=\frac{1}{2}|L 1 T\rangle$.

Equation (36) implies spinors are labeled by the $4 \times 4$ spin operator in the Dirac representation $\frac{i}{2} \gamma_{1} \gamma_{2}$, and the weak $\mathrm{SU}(\mathrm{L})_{L} \tau_{3}$ component. While most of the results in the paper are representation independent, a unitary transformation may be applied to the $(7+1)$-dimensional matrices to show the conventional-basis description used in Eq. (36). Indeed, $\frac{i}{2} P_{L} \gamma_{1} \gamma_{2}$ has a Dirac form with the unitary transformation $\gamma_{D}^{\mu}=U_{D}^{\dagger} \gamma^{\mu} U_{D}$ with $U_{D}=$ $\frac{1}{\sqrt{2}}\left(1-\gamma^{1} \gamma^{3}\right) \gamma^{0} \gamma^{2} \gamma^{3}$ (actually, it exchanges $\gamma^{1}$ and $\gamma^{3}$ ) and $|L 1 T\rangle$ is represented, after the unitary transformation $U_{D}^{\dagger}$, by $(0,0,0,0,0,0,0,0,0,0,0,0,0,0, i, 0)$. Next, we write all the conventional-basis states in this basis, and their association to the spin-extended basis states, with corresponding quantum numbers (notation used in Table IV and Ref. [7], written inbetween):

$(0,0,0,0,0,0,0,0,0,0,0,0,0,0, i, 0) \leftrightarrow T^{1}{ }_{L}, U^{1}{ }_{1 L}$

$(0,0,-i, 0,0,0,0,0,0,0,0,0,0,0,0,0) \leftrightarrow T^{2}{ }_{L}, U^{2}{ }_{1 L}$

$(0,0,0,0,0,0,-i, 0,0,0,0,0,0,0,0,0) \leftrightarrow T^{1}{ }_{R}, U^{1}{ }_{1 R}$

$(0,0,0,0,0,0,0,0,0,0, i, 0,0,0,0,0) \leftrightarrow T^{2}{ }_{R}, U^{2}{ }_{1 R}$

$(0,0,0,0,0,0,0,0,0,0,0,0,0,0,0,-i) \leftrightarrow B^{1}{ }_{L}, D^{1}{ }_{1 L}$

$(0,0,0, i, 0,0,0,0,0,0,0,0,0,0,0,0) \leftrightarrow B^{2}{ }_{L}, D^{2}{ }_{1 L}$

$(0,0,0,0,0,0,0, i, 0,0,0,0,0,0,0,0) \leftrightarrow B^{2}{ }_{R}, D^{2}{ }_{1 R}$

$(0,0,0,0,0,0,0,0,0,0,0,-i, 0,0,0,0) \leftrightarrow B^{2}{ }_{R}, D^{2}{ }_{1 R}$,

(A11)

where the spin-basis states are shown in extenso in Tables II and III.

For the fermion wave functions $\psi_{q h}^{\alpha}(x)$, we use polar coordinates, where the conventional and spin terms contain, respectively,

$\psi_{q h}^{\alpha}(x) \exp \left[i p^{\alpha}{ }_{q h}(x)\right] \leftrightarrow \psi_{q h}^{\alpha}(x) \exp \left[i c_{q h}^{\alpha}(x)\right], \quad$ for quarks $q=t, b$, with spin components $\alpha=1,2$, and chirality $h=L, R$. The magnitude part can be shown to be the same for both cases, as can be derived by comparing, e.g., the mass term. The vectors $W_{\mu}^{a}(x)$, $B_{\mu}(x)$ are real fields.

The phases appear in each term in both bases. For example, for the conventional basis and for the two polarizations $t_{L}(x)=\left(\begin{array}{l}\psi_{t L}^{1}(x) \\ \psi_{t L}^{2}(x)\end{array}\right)$ within the left-handed hypercharge $Y=1 / 3 \mathrm{SU}(2)_{L}$-doublet, we use the association 


$$
t_{L}(x) \rightarrow \psi_{t L}{ }_{t L}(x) \exp \left[i p^{1}{ }_{t L}(x)\right] U_{D}\left(\begin{array}{c}
0 \\
0 \\
0 \\
0 \\
0 \\
0 \\
0 \\
0 \\
0 \\
0 \\
0 \\
0 \\
0 \\
i \\
0
\end{array}\right)+\psi_{t L}^{2}(x) \exp \left[i p^{2}{ }_{t L}(x)\right] U_{D}\left(\begin{array}{c}
0 \\
0 \\
-i \\
0 \\
0 \\
0 \\
0 \\
0 \\
0 \\
0 \\
0 \\
0 \\
0 \\
0
\end{array}\right) \text {, }
$$

with $U_{D}$ applied to transform back from the Dirac representation, and we used the terms in Eqs. (A4) and (A5); for the spin basis, $\Psi_{t L}(x)=\boldsymbol{\psi}^{1}{ }_{t L}(x) \exp \left[i c^{1}{ }_{t L}(x)\right] T_{L}^{1}+\boldsymbol{\psi}^{2}{ }_{t L}(x) \exp \left[i c^{2}{ }_{t L}(x)\right] T_{L}^{2}$.

The Lagrangians' identity is shown, by checking that the same terms are reproduced in both bases, and finding independent constant phases that connect the two representations. In the following, we present the fermion-vector $\mathcal{L}_{F V}$ Lagrangian components: interactive (weak and hypercharge), kinetic, and also the fermion-scalar (Yukawa) $\mathcal{L}_{S F}$ Lagrangian. The subtitle contains the two-basis Lagrangian expressions in a concise notation, and then one component is given in an expanded form; the equations that link the phases in the two representations are written as they derive from the terms.

1. Weak

$$
\begin{aligned}
& \overline{\mathbf{q}}_{L}(x) \frac{1}{2} g \tau^{a} W_{\mu}^{a}(x) \gamma^{\mu} \mathbf{q}_{L}(x) \leftrightarrow \operatorname{tr}\left\{\boldsymbol{\Psi}_{q L}(x)^{\dagger} g I_{a} W_{\mu}^{a}(x) \gamma^{0} \gamma^{\mu} \boldsymbol{\Psi}_{q L}(x)\right\} \\
& \frac{g}{2}\left(\left(W^{3}{ }_{3}(x)-W_{0}^{3}(x)\right) \boldsymbol{\psi}^{1}{ }_{b L}(x)^{2}-2\left(\left(\cos \left(p^{1}{ }_{b L}(x)-p^{1}{ }_{t L}(x)\right) W^{1}{ }_{0}(x)\right.\right.\right. \\
& \left.-\cos \left(p^{1}{ }_{b L}(x)-p^{1}{ }_{t L}(x)\right) W^{1}{ }_{3}(x)+\sin \left(p^{1}{ }_{b L}(x)-p^{1}{ }_{t L}(x)\right)\left(W^{2}{ }_{0}(x)-W^{2}{ }_{3}(x)\right)\right) \boldsymbol{\psi}^{1}{ }_{t L}(x) \\
& +\left(\sin \left(p^{1}{ }_{b L}(x)-p^{2}{ }_{b L}(x)\right) W^{3}{ }_{2}(x)-\cos \left(p^{1}{ }_{b L}(x)-p^{2}{ }_{b L}(x)\right) W^{3}{ }_{1}(x)\right) \psi_{b L}{ }_{b L}(x) \\
& -\left(\cos \left(p^{1}{ }_{b L}(x)-p^{2}{ }_{t L}(x)\right) W^{1}{ }_{1}(x)-\left(\sin \left(p^{1}{ }_{b L}(x)-p^{2}{ }_{t L}(x)\right) W^{1}{ }_{2}(x)\right.\right. \\
& \left.\left.+\sin \left(p^{1}{ }_{b L}(x)-p^{2}{ }_{t L}(x)\right) W^{2}{ }_{1}(x)+\cos \left(p^{1}{ }_{b L}(x)-p^{2}{ }_{t L}(x)\right) W^{2}{ }_{2}(x)\right) \boldsymbol{\psi}_{t L}{ }_{t L}(x)\right) \boldsymbol{\psi}^{1}{ }_{b L}(x) \\
& +W^{3}{ }_{0}(x) \boldsymbol{\psi}^{1}{ }_{t L}(x)^{2}-W^{3}{ }_{3}(x) \boldsymbol{\psi}^{1}{ }_{t L}(x)^{2}-\left(W^{3}{ }_{0}(x)+W^{3}{ }_{3}(x)\right) \boldsymbol{\psi}_{b L}{ }_{b L}(x)^{2} \\
& +W^{3}{ }_{0}(x) \boldsymbol{\psi}^{2}{ }_{t L}(x)^{2}+W^{3}{ }_{3}(x) \boldsymbol{\psi}^{2}{ }_{t L}(x)^{2} \\
& -2 \cos \left(p^{1}{ }_{t L}(x)-p^{2}{ }_{t L}(x)\right) W^{3}{ }_{1}(x) \boldsymbol{\psi}^{1}{ }_{t L}(x) \boldsymbol{\psi}^{2}{ }_{t L}(x) \\
& +2 \sin \left(p^{1}{ }_{t L}(x)-p^{2}{ }_{t L}(x)\right) W^{3}{ }_{2}(x) \psi^{1}{ }_{t L}(x) \psi^{2}{ }_{t L}(x) \\
& -2 \boldsymbol{\psi}_{b L}^{2}(x)\left(\left(\cos \left(p^{2}{ }_{b L}(x)-p^{2}{ }_{t L}(x)\right) W^{1}{ }_{0}(x)+\cos \left(p^{2}{ }_{b L}(x)-p^{2}{ }_{t L}(x)\right) W^{1}{ }_{3}(x)\right.\right. \\
& \left.+\sin \left(p^{2}{ }_{b L}(x)-p^{2}{ }_{t L}(x)\right)\left(W^{2}{ }_{0}(x)+W^{2}{ }_{3}(x)\right)\right) \psi^{2}{ }_{t L}(x) \\
& -\left(\cos \left(p^{1}{ }_{t L}(x)-p^{2}{ }_{b L}(x)\right) W^{1}{ }_{1}(x)-\sin \left(p^{1}{ }_{t L}(x)-p^{2}{ }_{b L}(x)\right) W^{1}{ }_{2}(x)\right. \\
& \left.\left.-\sin \left(p^{1}{ }_{t L}(x)-p^{2}{ }_{b L}(x)\right)\left(W^{2}{ }_{1}(x)-\cos \left(p^{1}{ }_{t L}(x)-p^{2}{ }_{b L}(x)\right) W^{2}{ }_{2}(x)\right) \psi^{1}{ }_{t L}(x)\right)\right) \text {. }
\end{aligned}
$$

Comparing the corresponding expression in the spin basis, we derive the following phase relations (which retroactively provide such an expression): 


$$
\begin{gathered}
c^{1}{ }_{b L}(x)=p_{W 1}+p^{1}{ }_{b L}(x)+\frac{\pi}{2} \\
c^{1}{ }_{t L}(x)=p_{W 2}+p^{1}{ }_{t L}(x)+\pi \\
c^{2}{ }_{b L}(x)=p_{W 3}+p^{2}{ }_{b L}(x)+\frac{\pi}{2} \\
c^{2}{ }_{t L}(x)=p_{W 4}+p^{2}{ }_{t L}(x)+\pi \\
c^{2}{ }_{b R}(x)=p_{Z 1}+p^{2}{ }_{b R}(x) \\
c^{2}{ }_{t R}(x)=p_{Z 2}+p^{2}{ }_{t R}(x) \\
c^{1}{ }_{b R}(x)=p_{Z 1}+p^{1}{ }_{b R}(x)+\pi \\
c^{1}{ }_{t R}(x)=p_{Z 2}+p^{1}{ }_{t R}(x)+\pi,
\end{gathered}
$$

for arbitrary real constants $p_{W 1}, p_{W 2}, p_{W 3}, p_{W 4}, p_{Z 1}, p_{Z 2}$, requiring the identities $p_{W 1}=p_{W 3}=p_{W 4}=p_{W 2}$.

\section{Hypercharge}

$\overline{\mathbf{q}}_{L}(x) \frac{1}{6} g^{\prime} B_{\mu}(x) \gamma^{\mu} \mathbf{q}_{L}(x)+\bar{t}_{R}(x)\left[\frac{2}{3} g^{\prime} B_{\mu}(x)\right] \gamma^{\mu} t_{R}(x)+\bar{b}_{R}(x)\left[-\frac{1}{3} g^{\prime} B_{\mu}(x)\right] \gamma^{\mu} b_{R}(x) \leftrightarrow \operatorname{tr}\left\{\boldsymbol{\Psi}_{q L}(x)^{\dagger} \frac{1}{2} g^{\prime} Y_{o} B_{\mu}(x) \gamma^{0} \gamma^{\mu} \boldsymbol{\Psi}_{q L}(x)+\right.$ $\left.\Psi_{t R}^{\dagger}(x) \frac{1}{2} g^{\prime} Y_{o} B_{\mu}(x) \gamma^{0} \gamma^{\mu} \Psi_{t R}(x)+\Psi_{b R}^{\dagger}(x) \frac{1}{2} g^{\prime} Y_{o} B_{\mu}(x) \gamma^{0} \gamma^{\mu} \Psi_{b R}(x)\right\}$

$$
\begin{aligned}
& \frac{g^{\prime}}{6}\left(\left(B_{0}(x)-B_{3}(x)\right) \psi_{b L}^{1}(x)^{2}-2\left(\cos \left(p^{1}{ }_{b L}(x)-p^{2}{ }_{b L}(x)\right) B_{1}(x)\right.\right. \\
& \left.-\sin \left(p^{1}{ }_{b L}(x)-p_{b L}^{2}(x)\right) B_{2}(x)\right) \boldsymbol{\psi}_{b L}^{2}(x) \boldsymbol{\psi}_{b L}^{1}(x)-2 B_{0}(x) \boldsymbol{\psi}_{b R}^{1}(x)^{2}-2 B_{3}(x) \boldsymbol{\psi}_{b R}^{1}(x)^{2} \\
& +B_{0}(x) \psi^{1}{ }_{t L}(x)^{2}-B_{3}(x) \psi^{1}{ }_{t L}(x)^{2}+4 B_{0}(x) \psi^{1}{ }_{t R}(x)^{2}+4 B_{3}(x) \psi^{1}{ }_{t R}(x)^{2}+\left(B_{0}(x)+B_{3}(x)\right) \psi^{2}{ }_{b L}(x)^{2} \\
& -2 B_{0}(x) \boldsymbol{\psi}_{b R}^{2}(x)^{2}+2 B_{3}(x) \psi_{b R}^{2}(x)^{2}+B_{0}(x) \boldsymbol{\psi}_{t L}^{2}(x)^{2}+B_{3}(x) \boldsymbol{\psi}_{t L}^{2}(x)^{2}+4 B_{0}(x) \boldsymbol{\psi}_{t R}^{2}(x)^{2}-4 B_{3}(x){\boldsymbol{\psi}^{2}}_{t R}(x)^{2} \\
& +4 \cos \left(p_{b R}^{1}(x)-p_{b R}^{2}(x)\right) B_{1}(x) \boldsymbol{\psi}_{b R}^{1}(x) \boldsymbol{\psi}_{b R}^{2}(x)-4 \sin \left(p_{b R}^{1}(x)-p_{b R}^{2}(x)\right) B_{2}(x) \boldsymbol{\psi}_{b R}^{1}(x) \boldsymbol{\psi}_{b R}{ }_{b R}(x) \\
& -2 \cos \left(p^{1}{ }_{t L}(x)-p^{2}{ }_{t L}(x)\right) B_{1}(x) \psi^{1}{ }_{t L}(x) \psi^{2}{ }_{t L}(x)+2 \sin \left(p^{1}{ }_{t L}(x)-p^{2}{ }_{t L}(x)\right) B_{2}(x) \boldsymbol{\psi}^{1}{ }_{t L}(x) \boldsymbol{\psi}^{2}{ }_{t L}(x) \\
& \left.-8 \cos \left(p^{1}{ }_{t R}(x)-p^{2}{ }_{t R}(x)\right) B_{1}(x) \psi^{1}{ }_{t R}(x) \boldsymbol{\psi}^{2}{ }_{t R}(x)+8 \sin \left(p^{1}{ }_{t R}(x)-p^{2}{ }_{t R}(x)\right) B_{2}(x) \boldsymbol{\psi}^{1}{ }_{t R}(x) \boldsymbol{\psi}^{2}{ }_{t R}(x)\right) \text {. }
\end{aligned}
$$

\section{Kinetic}

$\overline{\mathbf{q}}_{L}(x) i \frac{1}{2} \stackrel{\leftrightarrow}{\partial}_{\mu} \gamma^{\mu} \mathbf{q}_{L}(x)+\bar{t}_{R}(x) i \frac{1}{2}{\stackrel{\leftrightarrow}{\partial_{\mu}}}^{\mu} t_{R}(x)+\bar{b}_{R}(x) i \frac{1}{2}{\stackrel{\leftrightarrow}{\partial_{\mu}}}^{\mu} b_{R}(x) \leftrightarrow \operatorname{tr} \frac{1}{2}\left\{\boldsymbol{\Psi}_{q L}^{\dagger}(x) i \stackrel{\leftrightarrow}{\partial_{\mu}} \gamma^{0} \gamma^{\mu} \boldsymbol{\Psi}_{q L}(x)+\Psi_{t R}^{\dagger}(x) i \stackrel{\leftrightarrow}{\partial_{\mu}} \gamma^{0} \gamma^{\mu} \Psi_{t R}(x)+\right.$ $\left.\Psi_{b R}^{\dagger}(x) i \stackrel{\leftrightarrow}{\partial_{\mu}} \gamma^{0} \gamma^{\mu} \Psi_{b R}(x)\right\}$

Using the fields' integrability property (belonging to Hilbert space), integration by parts has been applied to make the derivative substitution $i \partial_{\mu} \rightarrow i \frac{1}{2} \partial_{\mu}$. 


$$
\begin{aligned}
& -\psi^{2}{ }_{t L}(x)^{2}\left(\partial_{z} p^{2}{ }_{t L}(x)\right)+\psi^{2}{ }_{t R}(x)^{2}\left(\partial_{z} p^{2}{ }_{t R}(x)\right)-2 \sin \left[p^{1}{ }_{b R}(x)-p^{2}{ }_{b R}(x)\right] \psi^{1}{ }_{b R}(x) \psi_{b R}^{2}(x)\left(\partial_{y} p^{1}{ }_{b R}(x)\right) \\
& -2 \sin \left[p_{b R}^{1}(x)-p_{b R}^{2}(x)\right] \boldsymbol{\psi}_{b R}^{1}(x) \boldsymbol{\psi}_{b R}^{2}(x)\left(\partial_{y} p_{b R}^{2}(x)\right)+2 \cos \left[p_{b R}^{1}(x)-p_{b R}^{2}(x)\right] \boldsymbol{\psi}_{b R}^{2}(x)\left(\partial_{y} \boldsymbol{\psi}_{b R}^{1}(x)\right) \\
& +\cos \left[p^{1}{ }_{t L}(x)-p^{2}{ }_{t L}(x)\right] \psi^{2}{ }_{t L}(x)\left(\partial_{y} \psi^{1}{ }_{t L}(x)\right)+\cos \left[p^{1}{ }_{t R}(x)-p^{2}{ }_{t R}(x)\right] \boldsymbol{\psi}^{2}{ }_{t R}(x)\left(\partial_{y} \boldsymbol{\psi}^{1}{ }_{t R}(x)\right) \\
& -2 \cos \left[p^{1}{ }_{b R}(x)-p^{2}{ }_{b R}(x)\right] \boldsymbol{\psi}_{b R}^{1}(x)\left(\partial_{y} \boldsymbol{\psi}_{b R}^{2}(x)\right)+2 \cos \left[p_{b R}^{1}(x)-p_{b R}^{2}(x)\right] \boldsymbol{\psi}_{b R}^{1}(x) \boldsymbol{\psi}_{b R}^{2}(x)\left(\partial_{x} p^{1}{ }_{b R}(x)\right) \\
& +2 \cos \left[p_{b R}^{1}(x)-p_{b R}^{2}(x)\right] \boldsymbol{\psi}_{b R}^{1}(x) \boldsymbol{\psi}_{b R}^{2}(x)\left(\partial_{x} p_{b R}^{2}(x)\right)+2 \sin \left[p_{b R}^{1}(x)-p_{b R}^{2}(x)\right] \boldsymbol{\psi}_{b R}^{2}(x)\left(\partial_{x} \boldsymbol{\psi}_{b R}^{1}(x)\right) \\
& +\sin \left[p^{1}{ }_{t L}(x)-p^{2}{ }_{t L}(x)\right] \boldsymbol{\psi}^{2}{ }_{t L}(x)\left(\partial_{x} \boldsymbol{\psi}^{1}{ }_{t L}(x)\right)+\sin \left[p^{1}{ }_{t R}(x)-p^{2}{ }_{t R}(x)\right] \boldsymbol{\psi}^{2}{ }_{t R}(x)\left(\partial_{x} \boldsymbol{\psi}^{1}{ }_{t R}(x)\right) \\
& -2 \sin \left[p^{1}{ }_{b R}(x)-p^{2}{ }_{b R}(x)\right] \psi^{1}{ }_{b R}(x)\left(\partial_{x} \psi^{2}{ }_{b R}(x)\right)-\psi^{1}{ }_{t L}(x)\left(\cos \left[p^{1}{ }_{t L}(x)-p^{2}{ }_{t L}(x)\right]\left(\partial_{y} \psi^{2}{ }_{t L}(x)\right)\right. \\
& +\boldsymbol{\psi}^{2}{ }_{t L}(x)\left(\sin \left[p^{1}{ }_{t L}(x)-p^{2}{ }_{t L}(x)\right]\left(\partial_{y} p^{1}{ }_{t L}(x)\right)+\sin \left[p^{1}{ }_{t L}(x)-p^{2}{ }_{t L}(x)\right]\left(\partial_{y} p^{2}{ }_{t L}(x)\right)\right. \\
& \left.\left.-\cos \left[p^{1}{ }_{t L}(x)-p^{2}{ }_{t L}(x)\right]\left(\partial_{x} p^{1}{ }_{t L}(x)+\partial_{x} p^{2}{ }_{t L}(x)\right)\right)+\sin \left[p^{1}{ }_{t L}(x)-p^{2}{ }_{t L}(x)\right]\left(\partial_{x} \psi{ }_{t L}(x)\right)\right) \\
& -\boldsymbol{\psi}^{1}{ }_{t R}(x)\left(\cos \left[p^{1}{ }_{t R}(x)-p^{2}{ }_{t R}(x)\right]\left(\partial_{y} \boldsymbol{\psi}^{2}{ }_{t R}(x)\right)+\boldsymbol{\psi}^{2}{ }_{t R}(x)\left(\sin \left[p^{1}{ }_{t R}(x)-p^{2}{ }_{t R}(x)\right]\left(\partial_{y} p^{1}{ }_{t R}(x)\right)\right.\right. \\
& \left.+\sin \left[p^{1}{ }_{t R}(x)-p^{2}{ }_{t R}(x)\right]\left(\partial_{y} p^{2}{ }_{t R}(x)\right)-\cos \left[p^{1}{ }_{t R}(x)-p^{2}{ }_{t R}(x)\right]\left(\partial_{x} p^{1}{ }_{t R}(x)+\partial_{x} p^{2}{ }_{t R}(x)\right)\right) \\
& \left.+\sin \left[p^{1}{ }_{t R}(x)-p^{2}{ }_{t R}(x)\right]\left(\partial_{x} \psi^{2}{ }_{t R}(x)\right)\right)-2 \psi^{1}{ }_{b R}(x)^{2}\left(\partial_{t} p^{1}{ }_{b R}(x)\right) \\
& -\boldsymbol{\psi}^{1}{ }_{t L}(x)^{2}\left(-\left(\partial_{z} p^{1}{ }_{t L}(x)\right)+\partial_{t} p^{1}{ }_{t L}(x)\right)-\boldsymbol{\psi}^{1}{ }_{t R}(x)^{2}\left(\partial_{z} p^{1}{ }_{t R}(x)+\partial_{t} p^{1}{ }_{t R}(x)\right) \\
& -2 \psi_{b R}^{2}(x)^{2}\left(\partial_{t} p_{b R}^{2}(x)\right)-\psi^{2}{ }_{t L}(x)^{2}\left(\partial_{t} p^{2}{ }_{t L}(x)\right)-\psi^{2}{ }_{t R}(x)^{2}\left(\partial_{t} p^{2}{ }_{t R}(x)\right) \text {. }
\end{aligned}
$$

\section{Yukawa}

$\frac{\sqrt{2}}{v}\left[m_{t} \bar{t}_{R}(x) \tilde{\mathbf{H}}^{\dagger}(x) \mathbf{q}_{L}(x)+m_{b} \overline{\mathbf{q}}_{L}(x) \mathbf{H}(x) b_{R}(x)\right]+\{$ H.c. $\} \leftrightarrow \operatorname{tr} \frac{\sqrt{2}}{v}\left[m_{t} \Psi_{t R}^{\dagger}(x) \mathbf{H}_{t}(x) \boldsymbol{\Psi}_{q L}(x)+m_{b} \boldsymbol{\Psi}_{q L}^{\dagger}(x) \mathbf{H}_{b}(x) \Psi_{b R}(x)\right]+\{$ H.c. $\}$

The representation of scalars in the conventional and spin bases uses the association, e.g., $\mathbf{H} \gamma^{0}{ }_{4 \times 4} \rightarrow \mathbf{H}_{t}$; the conventional phases, written explicitly in Appendix B, are set to fit the spin basis, as both operators act equally on fermions, and we applied the gamma-matrix representation freedom of choice.

$$
\begin{aligned}
& \frac{1}{v}\left[\cos \left(p_{W 2}-p_{Z 1}-p_{\eta 1}(x)-p^{1}{ }_{b R}(x)+p^{1}{ }_{t L}(x)\right) m_{b} \eta^{r}{ }_{1}(x) \boldsymbol{\psi}^{1}{ }_{b R}(x) \psi^{1}{ }_{t L}(x)+\cos \left(p_{W 2}-p_{Z 2}-p_{\eta 0}(x)\right.\right. \\
& \left.+p^{1}{ }_{t L}(x)-p^{1}{ }_{t R}(x)\right) m_{t} \eta^{r}{ }_{0}(x) \boldsymbol{\psi}^{1}{ }_{t R}(x) \boldsymbol{\psi}^{1}{ }_{t L}(x)+\boldsymbol{\psi}^{1}{ }_{b L}(x)\left(\sin \left(p_{W 2}-p_{Z 2}+p_{\eta 1}(x)+p^{1}{ }_{b L}(x)-p^{1}{ }_{t R}(x)\right) m_{t} \eta^{r}{ }_{1}(x) \boldsymbol{\psi}^{1}{ }_{t R}(x)\right. \\
& \left.-\sin \left(p_{W 2}-p_{Z 1}+p_{\eta 0}(x)+p^{1}{ }_{b L}(x)-p^{1}{ }_{b R}(x)\right) m_{b} \eta^{r}{ }_{0}(x) \psi_{b R}^{1}(x)\right)-\cos \left(p_{W 2}-p_{Z 1}-p_{\eta 1}(x)-p_{b R}^{2}(x)\right. \\
& \left.+p^{2}{ }_{t L}(x)\right) m_{b} \eta^{r}{ }_{1}(x) \psi_{b R}^{2}(x) \psi_{t L}^{2}(x)-\cos \left(p_{W 2}-p_{Z 2}-p_{\eta 0}(x)+p^{2}{ }_{t L}(x)\right. \\
& \left.-p^{2}{ }_{t R}(x)\right) m_{t} \eta^{r}{ }_{0}(x) \psi_{t L}^{2}(x) \psi_{t R}^{2}(x)+\psi_{b L}^{2}(x)\left(\sin \left(p_{W 2}-p_{Z 1}+p_{\eta 0}(x)+p_{b L}^{2}(x)-p_{b R}^{2}(x)\right) m_{b} \eta^{r}{ }_{0}(x) \psi_{b R}^{2}(x)\right. \\
& \left.\left.-\sin \left(p_{W 2}-p_{Z 2}+p_{\eta 1}(x)+p^{2}{ }_{b L}(x)-p^{2}{ }_{t R}(x)\right) m_{t} \eta^{r}{ }_{1}(x) \psi^{2}{ }_{t R}(x)\right)\right] \text {, }
\end{aligned}
$$

requiring the identities $p_{Z 1}-\pi=p_{Z 2}-\frac{\pi}{2}=p_{W 1}$.

\section{APPENDIX B: SCALAR-VECTOR LAGRANGIAN $\mathcal{L}_{S V}$; CONJUGATE-HIGGS INVARIANCE}

For the scalar components, we also use expressions in polar coordinates, and in which the phase is written explicitly, to see its workings. Thus, for the conventional basis,

$$
\begin{gathered}
\mathbf{H}(x)=\frac{1}{\sqrt{2}}\left(\begin{array}{c}
\eta^{r}{ }_{1}(x) e^{i p^{1}{ }_{t}+i p_{\eta 1}(x)} \\
\eta^{r}{ }_{0}(x) e^{i p^{0}{ }^{0}+i p_{\eta 0}(x)}
\end{array}\right) \\
\tilde{\mathbf{H}}(x)=\frac{1}{\sqrt{2}}\left(\begin{array}{c}
-i \eta^{r}(x) e^{i p^{0}{ }_{b}-i p_{\eta 0}(x)} \\
i \eta^{r}{ }_{1}(x) e^{i p^{1}{ }_{b}-i p_{\eta 1}(x)}
\end{array}\right),
\end{gathered}
$$


where $p^{1}{ }_{b}, p^{1}{ }_{t}, p^{0}{ }_{b}, p^{0}{ }_{t}$ are charged and neutral phases, respectively, and $\overline{\mathbf{H}}_{\chi_{t}, \chi_{b}}(x)=\left(\chi_{t} \mathbf{H}(x), \chi_{b} \tilde{\mathbf{H}}(x)\right)$ is a $4 \times 4$ matrix, $\chi_{t}, \chi_{b}$ can be assumed to be real and their dependence in all terms is through the factor $\chi_{t}^{2}+\chi_{b}^{2}$, so their explicit form constitutes a likewise demonstration for $\mathcal{L}_{S V}$.

For the spin basis, we use a generalized expression for the scalar term with conjugated terms weighted by a multiplicative parameter $\lambda$, to keep track of terms, and with a normalization that makes $\mathcal{L}_{S V} \lambda$ independent:

$$
\begin{aligned}
\mathbf{H}_{a b}^{\mathrm{tot} \lambda}(x)= & \frac{1}{\sqrt{\left(1+\lambda^{2}\right.}}\left[\chi_{t}\left[\eta_{1}^{r}(x) e^{i \phi^{1}{ }_{t}+i p_{\eta 1}(x)}\left(\phi_{1}^{+}+\phi_{2}^{+}\right)+\eta_{0}^{r}(x) e^{i \phi^{0}{ }_{t}+i p_{\eta 0}(x)}\left(\phi_{1}^{0}+\phi_{2}^{0}\right)\right]+\chi_{b}\left[\eta_{1}^{r}(x) e^{i \phi^{1}{ }^{1}-i p_{\eta 1}(x)}\left(\phi_{1}^{+}-\phi_{2}^{+}\right)^{\dagger}\right.\right. \\
& \left.+\eta_{0}^{r}(x) e^{i \phi^{0}{ }_{b}-i p_{\eta 0}(x)}\left(\phi_{1}^{0}-\phi_{2}^{0}\right)^{\dagger}\right]+\lambda \chi_{t}\left[\eta_{1}^{r}(x) e^{i \phi^{1}{ }_{\lambda t}-i p_{\eta 1}(x)}\left(\phi_{1}^{+}+\phi_{2}^{+}\right)^{\dagger}+\eta_{0}^{r}(x) e^{i \phi^{0}{ }_{\lambda t}-i p_{\eta 0}(x)}\left(\phi_{1}^{0}+\phi_{2}^{0}\right)^{\dagger}\right] \\
& \left.+\lambda \chi_{b}\left[\eta_{1}^{r}(x) e^{i \phi^{1}{ }_{\lambda b}+i p_{\eta 1}(x)}\left(\phi_{1}^{+}-\phi_{2}^{+}\right)+\eta_{0}^{r}(x) e^{i \phi^{0}{ }_{\lambda b}+i p_{\eta 0}(x)}\left(\phi_{1}^{0}-\phi_{2}^{0}\right)\right]\right],
\end{aligned}
$$

where $\phi_{1,2}^{+}, \phi_{1,2}^{0}$ are defined in Table I, and $\phi^{1}{ }_{t}, \phi^{1}{ }_{b}, \phi^{0}{ }_{t}, \phi^{0}{ }_{b}$ are charged and neutral phases, respectively, and those with $\lambda$ correspond to the Hermitian-conjugate function [see Eqs. (50), (51)]. Given the chiral nature of the scalar components, they do not mix with their Hermitian-conjugate components.

Thus, $\mathcal{L}_{S V}=\mathbf{H}^{\dagger}(x) \mathbf{F}^{\mu \dagger}(x) \mathbf{F}_{\mu}(x) \mathbf{H}(x)$, with $\mathbf{F}_{\mu}(x)=i \partial_{\mu}+\frac{1}{2} g \boldsymbol{\tau} \cdot \mathbf{W}_{\mu}(x)+\frac{1}{2} g^{\prime} B_{\mu}(x), \mathbf{W}_{\mu}(x)=\left(W_{\mu}^{1}(x), W_{\mu}^{2}(x), W_{\mu}^{3}(x)\right)$ (cf. Eqs. (42) and (43)) is compared with $\frac{1}{2} \operatorname{tr}\left\{\left[\mathbf{F}^{\prime \prime}(x), \mathbf{H}_{a b}^{\mathrm{tot} \lambda}(x)\right]_{ \pm}^{\dagger}\left[\mathbf{F}^{\prime \prime}(x), \mathbf{H}_{a b}^{\operatorname{tot} \lambda}(x)\right]_{ \pm}\right\}_{\text {sym }}$, where $\mathbf{F}^{\prime \prime}(x)=\left[i \partial_{\mu}+g W_{\mu}^{i}(x) I_{i}+\right.$ $\left.\frac{1}{2} g^{\prime} B_{\mu}(x) Y_{o}\right] \gamma_{0} \gamma^{\mu}$; the subindex sym means only symmetric $\gamma_{\mu} \gamma_{\nu}$ components are taken.

\section{Square W}

$\operatorname{tr} \overline{\mathbf{H}}^{\dagger}(x) \frac{1}{2} g \boldsymbol{\tau} \cdot \mathbf{W}_{\mu}(x) \frac{1}{2} g \boldsymbol{\tau} \cdot \mathbf{W}^{\mu}(x) \overline{\mathbf{H}}(x) \leftrightarrow \frac{1}{2} \operatorname{tr}\left\{\left[g W_{0}^{n}(x) I_{n} \gamma_{0} \gamma^{0}, \mathbf{H}_{a b}^{\text {toth }}(x)\right]^{\dagger}\left[g W_{0}^{m}(x) I_{m} \gamma_{0} \gamma^{0}, \mathbf{H}_{a b}^{\operatorname{tot} \lambda}(x)\right]+\left\{g W_{j}^{n}(x) I_{n} \gamma_{0} \gamma^{j}\right.\right.$, $\left.\left.\mathbf{H}_{a b}^{\mathrm{tot \lambda}}(x)\right\}^{\dagger}\left\{g W_{k}^{m}(x) I_{m} \gamma_{0} \gamma^{k}, \mathbf{H}_{a b}^{\mathrm{tot} \lambda}(x)\right\}\right\}$

$$
\frac{1}{8} g^{2}\left(\chi_{t}^{2}+\chi_{b}^{2}\right)\left(\eta_{0}^{r}(x)^{2}+\eta_{1}^{r}(x)^{2}\right) W_{\mu}^{n}(x) W^{n \mu}(x) .
$$

\section{Square B}

$\operatorname{tr} \overline{\mathbf{H}}^{\dagger}(x) \frac{1}{2} g^{\prime} B_{\mu}(x) \frac{1}{2} g^{\prime} B^{\mu}(x) \overline{\mathbf{H}}(x) \leftrightarrow \frac{1}{2} \operatorname{tr}\left\{\left[\frac{1}{2} g^{\prime} B_{0}(x) Y_{o} \gamma_{0} \gamma^{0}, \mathbf{H}_{a b}^{\text {tot } \lambda}(x)\right]^{\dagger}\left[\frac{1}{2} g^{\prime} B_{0}(x) Y_{o} \gamma_{0} \gamma^{0}, \mathbf{H}_{a b}^{\text {tot } \lambda}(x)\right]+\left\{\frac{1}{2} g^{\prime} B_{j}(x) Y_{o} \gamma_{0} \gamma^{j}\right.\right.$, $\left.\left.\mathbf{H}_{a b}^{\operatorname{tot} \lambda}(x)\right\}^{\dagger}\left\{\frac{1}{2} g^{\prime} B_{k}(x) Y_{o} \gamma_{0} \gamma^{k}, \mathbf{H}_{a b}^{\mathrm{tot} \lambda}(x)\right\}\right\}$

$$
\frac{1}{8} g^{\prime 2}\left(\chi_{t}^{2}+\chi_{b}^{2}\right)\left(\eta_{0}^{r}(x)^{2}+\eta_{1}^{r}(x)^{2}\right) B_{\mu}(x) B^{\mu}(x)
$$

\section{Cross B-W}

$\operatorname{tr}\left\{\overline{\mathbf{H}}^{\dagger}(x) \frac{1}{2} g \boldsymbol{\tau} \cdot \mathbf{W}_{\mu}(x) \frac{1}{2} g^{\prime} B^{\mu}(x) \overline{\mathbf{H}}(x)+\overline{\mathbf{H}}^{\dagger}(x) \frac{1}{2} g^{\prime} B^{\mu}(x) \frac{1}{2} g \boldsymbol{\tau} \cdot \mathbf{W}_{\mu}(x) \overline{\mathbf{H}}(x)\right\} \leftrightarrow \frac{1}{2} \operatorname{tr}\left\{\left[\frac{1}{2} g^{\prime} B_{0}(x) Y_{o} \gamma_{0} \gamma^{0}, \mathbf{H}_{a b}^{\operatorname{tot} \lambda}(x)\right]^{\dagger}\left[g W_{0}^{n}(x) I_{n} \gamma_{0} \gamma^{0}\right.\right.$, $\left.\mathbf{H}_{a b}^{\mathrm{tot} \lambda}(x)\right]+\left[g W_{0}^{n}(x) I_{n} \gamma_{0} \gamma^{0}, \mathbf{H}_{a b}^{\mathrm{tot} \lambda}(x)\right]^{\dagger}\left[\frac{1}{2} g^{\prime} B_{0}(x) Y_{o} \gamma_{0} \gamma^{0}, \mathbf{H}_{a b}^{\mathrm{tot} \lambda}(x)\right]+\left\{\frac{1}{2} g^{\prime} B_{j}(x) Y_{o} \gamma_{0} \gamma^{j}, \mathbf{H}_{a b}^{\mathrm{tot} \lambda}(x)\right\}^{\dagger}\left\{g W_{k}^{n}(x) I_{n} \gamma_{0} \gamma^{k}, \mathbf{H}_{a b}^{\mathrm{tot} \lambda}(x)\right\}+$ $\left.\left\{g W_{j}^{n}(x) I_{n} \gamma_{0} \gamma^{j}, \mathbf{H}_{a b}^{\operatorname{tot} \lambda}(x)\right\}^{\dagger}\left\{\frac{1}{2} g^{\prime} B_{k}(x) Y_{o} \gamma_{0} \gamma^{k}, \mathbf{H}_{a b}^{\text {tot } \lambda}(x)\right\}\right\}$

$$
\begin{aligned}
& \frac{1}{4} g g^{\prime}\left(\chi_{t}^{2}+\chi_{b}^{2}\right)\left(-B_{0}(x) W^{3}{ }_{0}(x) \eta^{r}{ }_{0}(x)^{2}+B_{1}(x) W^{3}{ }_{1}(x) \eta^{r}{ }_{0}(x)^{2}+B_{2}(x) W^{3}{ }_{2}(x) \eta^{r}{ }_{0}(x)^{2}+B_{3}(x) W^{3}{ }_{3}(x) \eta^{r}{ }_{0}(x)^{2}\right. \\
& \quad+2 \cos \left[p^{0}{ }_{t}-p^{1}{ }_{b}-p_{\eta 0}(x)+p_{\eta 1}(x)\right] B_{0}(x) W^{1}{ }_{0}(x) \eta^{r}{ }_{0}(x) \eta^{r}{ }_{1}(x)-2 \sin \left[p^{0}{ }_{t}-p^{1}{ }_{b}-p_{\eta 0}(x)+p_{\eta 1}(x)\right] B_{0}(x) W^{2}{ }_{0}(x) \eta^{r}{ }_{0}(x) \eta^{r}{ }_{1}(x) \\
& -2 \cos \left[p^{0}{ }_{t}-p^{1}{ }_{b}-p_{\eta 0}(x)+p_{\eta 1}(x)\right] B_{1}(x) W^{1}{ }_{1}(x) \eta^{r}{ }_{0}(x) \eta^{r}{ }_{1}(x)+2 \sin \left[p^{0}{ }_{t}-p^{1}{ }_{b}-p_{\eta 0}(x)+p_{\eta 1}(x)\right] B_{1}(x) W^{2}{ }_{1}(x) \eta^{r}{ }_{0}(x) \eta^{r}{ }_{1}(x) \\
& -2 \cos \left[p^{0}{ }_{t}-p^{1}{ }_{b}-p_{\eta 0}(x)+p_{\eta 1}(x)\right] B_{2}(x) W^{1}{ }_{2}(x) \eta^{r}{ }_{0}(x) \eta^{r}{ }_{1}(x)+2 \sin \left[p^{0}{ }_{t}-p^{1}{ }_{b}-p_{\eta 0}(x)+p_{\eta 1}(x)\right] B_{2}(x) W^{2}{ }_{2}(x) \eta^{r}{ }_{0}(x) \eta^{r}{ }_{1}(x) \\
& -2 \cos \left[p^{0}{ }_{t}-p^{1}{ }_{b}-p_{\eta 0}(x)+p_{\eta 1}(x)\right] B_{3}(x) W^{1}{ }_{3}(x) \eta^{r}{ }_{0}(x) \eta^{r}{ }_{1}(x)+2 \sin \left[p^{0}{ }_{t}-p^{1}{ }_{b}-p_{\eta 0}(x)+p_{\eta 1}(x)\right] B_{3}(x) W^{2}{ }_{3}(x) \eta^{r}{ }_{0}(x) \eta^{r}{ }_{1}(x) \\
& \\
& \left.+B_{0}(x) W^{3}{ }_{0}(x) \eta^{r}{ }_{1}(x)^{2}-B_{1}(x) W^{3}{ }_{1}(x) \eta^{r}{ }_{1}(x)^{2}-B_{2}(x) W^{3}{ }_{2}(x) \eta^{r}{ }_{1}(x)^{2}-B_{3}(x) W^{3}{ }_{3}(x) \eta^{r}{ }_{1}(x)^{2}\right) .
\end{aligned}
$$

\section{Cross W-derivative}

$\operatorname{tr}\left\{\overline{\mathbf{H}}^{\dagger}(x) \frac{1}{2} g \boldsymbol{\tau} \cdot \mathbf{W}_{\mu}(x) i \partial^{\mu} \overline{\mathbf{H}}(x)-\overline{\mathbf{H}}^{\dagger}(x) i \stackrel{\check{\partial}}{\mu} \frac{1}{2} g \boldsymbol{\tau} \cdot \mathbf{W}_{\mu}(x) \overline{\mathbf{H}}(x)\right\} \leftrightarrow \frac{1}{2} \operatorname{tr}\left\{\left[-i \check{\partial}^{0} \gamma_{0} \gamma^{0}, \mathbf{H}_{a b}^{\mathrm{tot} \lambda}(x)\right]^{\dagger}\left[g W_{0}^{n}(x) I_{n} \gamma_{0} \gamma^{0}, \mathbf{H}_{a b}^{\mathrm{tot} \lambda}(x)\right]+\right.$ $\left[g W_{0}^{n}(x) I_{n} \gamma_{0} \gamma^{0}, \mathbf{H}_{a b}^{\mathrm{tot} \lambda}(x)\right]^{\dagger}\left[i \partial_{0} \gamma_{0} \gamma^{0}, \mathbf{H}_{a b}^{\mathrm{tot} \lambda}(x)\right]+\left\{-i \overleftarrow{\partial}_{j} \gamma_{0} \gamma^{j}, \mathbf{H}_{a b}^{\mathrm{tot} \lambda}(x)\right\}^{\dagger}\left\{g W_{k}^{n}(x) I_{n} \gamma_{0} \gamma^{k}, \mathbf{H}_{a b}^{\mathrm{tot} \lambda}(x)\right\}+\left\{g W_{j}^{n}(x) I_{n} \gamma_{0} \gamma^{j}\right.$, $\left.\left.\mathbf{H}_{a b}^{\mathrm{tot} \lambda}(x)\right\}^{\dagger}\left\{i \partial_{k} \gamma_{0} \gamma^{k}, \mathbf{H}_{a b}^{\mathrm{tot}} \lambda(x)\right\}\right\}$ 
As for the kinetic term in $\mathcal{L}_{F V}$, the fields' integrability property leads to derivatives in the form $i \frac{1}{2} \stackrel{\leftrightarrow}{\partial_{\mu}}$; similarly for the cross B-derivative and d'Alembert terms next.

$$
\begin{aligned}
& \frac{1}{2} g\left(\chi_{t}^{2}+\chi_{b}^{2}\right)\left(\eta^{r}{ }_{1}(x)^{2}\left(W^{3}{ }_{3}(x)\left(\partial_{z} p_{\eta 1}(x)\right)+W^{3}{ }_{2}(x)\left(\partial_{y} p_{\eta 1}(x)\right)+W^{3}{ }_{1}(x)\left(\partial_{x} p_{\eta 1}(x)\right)-W^{3}{ }_{0}(x)\left(\partial_{t} p_{\eta 1}(x)\right)\right)\right. \\
& -\eta^{r}{ }_{1}(x)\left(W ^ { 2 } { } _ { 3 } ( x ) \left(\sin \left[p^{0}{ }_{b}-p^{1}{ }_{b}-p_{\eta 0}(x)+p_{\eta 1}(x)\right] \eta^{r}{ }_{0}(x)\left(\partial_{z} p_{\eta 0}(x)+\partial_{z} p_{\eta 1}(x)\right)\right.\right. \\
& \left.+\cos \left[p^{0}{ }_{b}-p^{1}{ }_{b}-p_{\eta 0}(x)+p_{\eta 1}(x)\right]\left(\partial_{z} \eta^{r}{ }_{0}(x)\right)\right) \\
& +W^{1}{ }_{3}(x)\left(-\cos \left[p^{0}{ }_{b}-p^{1}{ }_{b}-p_{\eta 0}(x)+p_{\eta 1}(x)\right] \eta^{r}{ }_{0}(x)\left(\partial_{z} p_{\eta 0}(x)+\partial_{z} p_{\eta 1}(x)\right)+\sin \left[p^{0}{ }_{b}-p^{1}{ }_{b}-p_{\eta 0}(x)+p_{\eta 1}(x)\right]\left(\partial_{z} \eta^{r}{ }_{0}(x)\right)\right) \\
& -\cos \left[p^{0}{ }_{b}-p^{1}{ }_{b}-p_{\eta 0}(x)+p_{\eta 1}(x)\right] W^{1}{ }_{2}(x) \eta^{r}{ }_{0}(x)\left(\partial_{y} p_{\eta 0}(x)\right)+\sin \left[p^{0}{ }_{b}-p^{1}{ }_{b}-p_{\eta 0}(x)+p_{\eta 1}(x)\right] W^{2}{ }_{2}(x) \eta^{r}{ }_{0}(x)\left(\partial_{y} p_{\eta 0}(x)\right) \\
& -\cos \left[p_{b}^{0}{ }_{b}-p^{1}{ }_{b}-p_{\eta 0}(x)+p_{\eta 1}(x)\right] W^{1}{ }_{2}(x) \eta^{r}{ }_{0}(x)\left(\partial_{y} p_{\eta 1}(x)\right)+\sin \left[p_{b}^{0}-p^{1}{ }_{b}-p_{\eta 0}(x)+p_{\eta 1}(x)\right] W^{2}{ }_{2}(x) \eta^{r}{ }_{0}(x)\left(\partial_{y} p_{\eta 1}(x)\right) \\
& +\sin \left[p^{0}{ }_{b}-p^{1}{ }_{b}-p_{\eta 0}(x)+p_{\eta 1}(x)\right] W^{1}{ }_{2}(x)\left(\partial_{y} \eta^{r}{ }_{0}(x)\right)+\cos \left[p^{0}{ }_{b}-p^{1}{ }_{b}-p_{\eta 0}(x)+p_{\eta 1}(x)\right] W^{2}{ }_{2}(x)\left(\partial_{y} \eta^{r}{ }_{0}(x)\right) \\
& -\cos \left[p^{0}{ }_{b}-p^{1}{ }_{b}-p_{\eta 0}(x)+p_{\eta 1}(x)\right] W^{1}{ }_{1}(x) \eta^{r}{ }_{0}(x)\left(\partial_{x} p_{\eta 0}(x)\right) \\
& +\sin \left[p_{b}^{0}{ }_{b}-p^{1}{ }_{b}-p_{\eta 0}(x)+p_{\eta 1}(x)\right] W^{2}{ }_{1}(x) \eta^{r}{ }_{0}(x)\left(\partial_{x} p_{\eta 0}(x)\right)-\cos \left[p^{0}{ }_{b}-p^{1}{ }_{b}-p_{\eta 0}(x)+p_{\eta 1}(x)\right] W^{1}{ }_{1}(x) \eta^{r}{ }_{0}(x)\left(\partial_{x} p_{\eta 1}(x)\right) \\
& +\sin \left[p^{0}{ }_{b}-p^{1}{ }_{b}-p_{\eta 0}(x)+p_{\eta 1}(x)\right] W^{2}{ }_{1}(x) \eta^{r}{ }_{0}(x)\left(\partial_{x} p_{\eta 1}(x)\right)+\sin \left[p^{0}{ }_{b}-p^{1}{ }_{b}-p_{\eta 0}(x)+p_{\eta 1}(x)\right] W^{1}{ }_{1}(x)\left(\partial_{x} \eta^{r}{ }_{0}(x)\right) \\
& +\cos \left[p^{0}{ }_{b}-p^{1}{ }_{b}-p_{\eta 0}(x)+p_{\eta 1}(x)\right] W^{2}{ }_{1}(x)\left(\partial_{x} \eta^{r}{ }_{0}(x)\right)+\cos \left[p^{0}{ }_{b}-p^{1}{ }_{b}-p_{\eta 0}(x)+p_{\eta 1}(x)\right] W^{1}{ }_{0}(x) \eta^{r}{ }_{0}(x)\left(\partial_{t} p_{\eta 0}(x)\right) \\
& -\sin \left[p_{b}^{0}{ }_{b}-p^{1}{ }_{b}-p_{\eta 0}(x)+p_{\eta 1}(x)\right] W^{2}{ }_{0}(x) \eta^{r}{ }_{0}(x)\left(\partial_{t} p_{\eta 0}(x)\right)+\cos \left[p^{0}{ }_{b}-p^{1}{ }_{b}-p_{\eta 0}(x)+p_{\eta 1}(x)\right] W^{1}{ }_{0}(x) \eta^{r}{ }_{0}(x)\left(\partial_{t} p_{\eta 1}(x)\right) \\
& -\sin \left[p^{0}{ }_{b}-p^{1}{ }_{b}-p_{\eta 0}(x)+p_{\eta 1}(x)\right] W^{2}{ }_{0}(x) \eta^{r}{ }_{0}(x)\left(\partial_{t} p_{\eta 1}(x)\right)-\sin \left[p^{0}{ }_{b}-p^{1}{ }_{b}-p_{\eta 0}(x)+p_{\eta 1}(x)\right] W^{1}{ }_{0}(x)\left(\partial_{t} \eta^{r}{ }_{0}(x)\right) \\
& \left.-\cos \left[p_{b}^{0}{ }_{b}-p^{1}{ }_{b}-p_{\eta 0}(x)+p_{\eta 1}(x)\right] W^{2}{ }_{0}(x)\left(\partial_{t} \eta^{r}{ }_{0}(x)\right)\right)+\eta^{r}{ }_{0}(x)\left(-W^{3}{ }_{3}(x) \eta^{r}{ }_{0}(x)\left(\partial_{z} p_{\eta 0}(x)\right)\right. \\
& +\sin \left[p^{0}{ }_{b}-p^{1}{ }_{b}-p_{\eta 0}(x)+p_{\eta 1}(x)\right] W^{1}{ }_{3}(x)\left(\partial_{z} \eta^{r}{ }_{1}(x)\right) \\
& +\cos \left[p^{0}{ }_{b}-p^{1}{ }_{b}-p_{\eta 0}(x)+p_{\eta 1}(x)\right] W^{2}{ }_{3}(x)\left(\partial_{z} \eta^{r}{ }_{1}(x)\right)-W^{3}{ }_{2}(x) \eta^{r}{ }_{0}(x)\left(\partial_{y} p_{\eta 0}(x)\right) \\
& +\sin \left[p^{0}{ }_{b}-p^{1}{ }_{b}-p_{\eta 0}(x)+p_{\eta 1}(x)\right] W^{1}{ }_{2}(x)\left(\partial_{y} \eta^{r}{ }_{1}(x)\right) \\
& +\cos \left[p^{0}{ }_{b}-p^{1}{ }_{b}-p_{\eta 0}(x)+p_{\eta 1}(x)\right] W^{2}{ }_{2}(x)\left(\partial_{y} \eta^{r}{ }_{1}(x)\right)-W^{3}{ }_{1}(x) \eta^{r}{ }_{0}(x)\left(\partial_{x} p_{\eta 0}(x)\right) \\
& +\sin \left[p^{0}{ }_{b}-p^{1}{ }_{b}-p_{\eta 0}(x)+p_{\eta 1}(x)\right] W^{1}{ }_{1}(x)\left(\partial_{x} \eta^{r}{ }_{1}(x)\right) \\
& +\cos \left[p^{0}{ }_{b}-p^{1}{ }_{b}-p_{\eta 0}(x)+p_{\eta 1}(x)\right] W^{2}{ }_{1}(x)\left(\partial_{x} \eta^{r}{ }_{1}(x)\right)+W^{3}{ }_{0}(x) \eta^{r_{0}}(x)\left(\partial_{t} p_{\eta 0}(x)\right) \\
& \left.\left.-\sin \left[p^{0}{ }_{b}-p^{1}{ }_{b}-p_{\eta 0}(x)+p_{\eta 1}(x)\right] W^{1}{ }_{0}(x)\left(\partial_{t} \eta^{r}{ }_{1}(x)\right)-\cos \left[p^{0}{ }_{b}-p^{1}{ }_{b}-p_{\eta 0}(x)+p_{\eta 1}(x)\right] W^{2}{ }_{0}(x)\left(\partial_{t} \eta^{r}{ }_{1}(x)\right)\right)\right) \text {, }
\end{aligned}
$$

from which one derives the phase connections

$$
\begin{array}{r}
\phi_{\lambda t}^{1}=p^{0}{ }_{t}-p^{1}{ }_{t}+\phi^{0}{ }_{\lambda t}-\frac{\pi}{2} \\
\phi^{1}{ }_{b}=p^{0}{ }_{t}-p^{1}{ }_{t}+\phi^{0}{ }_{b}-\frac{\pi}{2} \\
\phi^{1}{ }_{t}=p^{0}{ }_{b}-p^{1}{ }_{b}+\phi^{0}{ }_{t}+\frac{\pi}{2} \\
\phi_{\lambda b}^{1}=p^{0}{ }_{b}-p^{1}{ }_{b}+\phi^{0}{ }_{\lambda b}+\frac{\pi}{2} .
\end{array}
$$

\section{Cross B-derivative}

$\operatorname{tr}\left\{\overline{\mathbf{H}}^{\dagger}(x) \frac{1}{2} g^{\prime} B_{\mu}(x) i \partial^{\mu} \overline{\mathbf{H}}(x)-\overline{\mathbf{H}}^{\dagger}(x) i \check{\partial}^{\mu} \frac{1}{2} g^{\prime} B_{\mu}(x) \overline{\mathbf{H}}(x)\right\} \leftrightarrow \frac{1}{2} \operatorname{tr}\left\{\left[-i \overleftarrow{\partial}^{0} \gamma_{0} \gamma^{0}, \mathbf{H}_{a b}^{\mathrm{tot} \lambda}(x)\right]^{\dagger}\left[\frac{1}{2} g^{\prime} B_{0}(x) Y_{o} \gamma_{0} \gamma^{0}, \mathbf{H}_{a b}^{\mathrm{tot} \lambda}(x)\right]+\right.$ $\left[\frac{1}{2} g^{\prime} B_{0}(x) Y_{o} \gamma_{0} \gamma^{0}, \mathbf{H}_{a b}^{\mathrm{tot} \lambda}(x)\right]^{\dagger}\left[i \partial^{0} \gamma_{0} \gamma^{0}, \mathbf{H}_{a b}^{\mathrm{tot} \lambda}(x)\right]+\left\{-i \overleftarrow{\partial}_{j} \gamma_{0} \gamma^{j}, \mathbf{H}_{a b}^{\mathrm{tot} \lambda}(x)\right\}^{\dagger}\left\{\frac{1}{2} g^{\prime} B_{k}(x) Y_{o} \gamma_{0} \gamma^{k}, \mathbf{H}_{a b}^{\mathrm{tot} \lambda}(x)\right\}+\left\{\frac{1}{2} g^{\prime} B_{j}(x) Y_{o} \gamma_{0} \gamma^{j}\right.$, $\left.\left.\mathbf{H}_{a b}^{\mathrm{tot} \lambda}(x)\right\}^{\dagger}\left\{i \partial_{k} \gamma_{0} \gamma^{k}, \mathbf{H}_{a b}^{\mathrm{tot} \lambda}(x)\right\}\right\}$ 


$$
\begin{aligned}
& \frac{1}{2}\left(\chi_{b}^{2}+\chi_{t}^{2}\right) g^{\prime}\left(\left(B_{3}(x)\left(\partial_{z} p_{\eta 0}(x)\right)+B_{2}(x)\left(\partial_{y} p_{\eta 0}(x)\right)+B_{1}(x)\left(\partial_{x} p_{\eta 0}(x)\right)-B_{0}(x)\left(\partial_{t} p_{\eta 0}(x)\right)\right) \eta^{r}{ }_{0}(x)^{2}+\left(B_{3}(x)\left(\partial_{z} p_{\eta 1}(x)\right)\right.\right. \\
& \left.\left.\quad+B_{2}(x)\left(\partial_{y} p_{\eta 1}(x)\right)+B_{1}(x)\left(\partial_{x} p_{\eta 1}(x)\right)-B_{0}(x)\left(\partial_{t} p_{\eta 1}(x)\right)\right) \eta^{r}{ }_{1}(x)^{2}\right) .
\end{aligned}
$$

In addition to the above equations, we derive

$$
p^{0}{ }_{t}=-p^{0}{ }_{b}+p^{1}{ }_{b}+p^{1}{ }_{t}
$$

As the similarity transformation phases in e.g. $\mathbf{H}, \tilde{\mathbf{H}}$, this relation accounts for the sign change for complex conjugate components.

$$
\begin{aligned}
& \operatorname{tr} \overline{\mathbf{H}}^{\dagger}(x) \overleftarrow{\partial^{\mu}} \partial_{\mu} \overline{\mathbf{H}}(x) \leftrightarrow-\frac{1}{2} \operatorname{tr}\left\{\left[\overleftarrow{\partial_{0} \gamma_{0}} \gamma^{0}, \mathbf{H}_{a b}^{\mathrm{tot} \lambda}(x)\right]^{\dagger}\left[\partial_{0} \gamma_{0} \gamma^{0}, \mathbf{H}_{a b}^{\mathrm{tot} \lambda}(x)\right]-\left\{\overleftarrow{\partial_{j} \gamma_{0}} \gamma^{j}, \mathbf{H}_{a b}^{\mathrm{tot} \lambda}(x)\right\}^{\dagger}\left\{\partial_{k} \gamma_{0} \gamma^{k}, \mathbf{H}_{a b}^{\mathrm{tot} \lambda}(x)\right\}\right\} \\
& \frac{1}{2}\left(\chi_{b}^{2}+\chi_{t}^{2}\right)\left(\left(\partial_{z} \eta_{1}^{r}(x)\right)^{2}+\eta_{0}^{r}(x)^{2}\left(\partial_{z} p_{\eta 0}(x)\right)^{2}+\eta_{1}^{r}(x)^{2}\left(\partial_{z} p_{\eta 1}(x)\right)^{2}+\left(\partial_{z} \eta_{0}^{r}(x)\right)^{2}+\left(\partial_{y} \eta_{1}^{r}(x)\right)^{2}+\eta^{r}{ }_{0}(x)^{2}\left(\partial_{y} p_{\eta 0}(x)\right)^{2}\right. \\
& +\eta_{1}^{r}(x)^{2}\left(\partial_{y} p_{\eta 1}(x)\right)^{2}+\left(\partial_{y} \eta_{0}^{r}(x)\right)^{2}+\left(\partial_{x} \eta_{1}^{r}(x)\right)^{2}+\eta_{0}^{r}(x)^{2}\left(\partial_{x} p_{\eta 0}(x)\right)^{2}+\eta_{1}^{r}(x)^{2}\left(\partial_{x} p_{\eta 1}(x)\right)^{2}+\left(\partial_{x} \eta_{0}^{r}(x)\right)^{2}-\left(\partial_{t} \eta_{1}^{r}(x)\right)^{2} \\
& \left.-\eta^{r}{ }_{0}(x)^{2}\left(\partial_{t} p_{\eta 0}(x)\right)^{2}-\eta_{1}^{r}(x)^{2}\left(\partial_{t} p_{\eta 1}(x)\right)^{2}-\left(\partial_{t} \eta^{r}{ }_{0}(x)\right)^{2}\right)
\end{aligned}
$$

Each of the $\mathcal{L}_{S V}$ terms is indeed proportional to the combination $\chi_{b}^{2}+\chi_{t}^{2}$, which manifests the t-b symmetry of this component, as the phases that connect the two representations were obtained.

We thus completed the demonstration of the SM Lagrangian terms' equivalence in two bases; we conclude the spin-space representation reproduces the same properties of SM generators.

[1] S. L. Glashow, Nucl. Phys. 22, 579 (1961); S. Weinberg, Phys. Rev. Lett. 19, 1264 (1967); A. Salam, in Elementary Particle Theory, edited by W. Svartholm (Almquist and Wiskell, Stockholm, 1968), p. 367.

[2] F. Englert and R. Brout, Phys. Rev. Lett. 13, 321 (1964).

[3] P. W. Higgs, Phys. Lett. 12. 132 (1964).

[4] K. A. Olive et al. (Particle Data Group), Chin. Phys. C 38, 090001 (2014).

[5] G. Aad et al. (ATLAS Collaboration), Phys. Rev. Lett. 108, 111803 (2012); S. Chatrchyan et al. (CMF Collaboration), Phys. Rev. Lett. 108, 111804 (2012).

[6] J. Besprosvany, Int. J. Theor. Phys. 39, 2797 (2000); Nuc. Phys. B (Proc. Suppl.) 101, 323 (2001); J. Besprosvany and R. Romero, AIP Conf. Proc., 1323, 16 (2010).

[7] J. Besprosvany and R. Romero, Int. J. Mod. Phys. A 29, 1450144 (2014).

[8] J. Besprosvany, Int. J. Mod. Phys. A 20, 77 (2005).

[9] R. Romero and J. Besprosvany, arXiv:1611.07446v1.

[10] J. Besprosvany, Phys. Lett. B 578, 181 (2004).

[11] L. B. Landau and E. M. Lifshitz, Quantum Mechanics (Pergamon, London, 1965).

[12] F. lachello and I. Talmi, Rev. Mod. Phys. 59, 339 (1987).

[13] J. Bardeen, L. N. Cooper, and J. R. Schrieffer, Phys. Rev. 108, 1175 (1957).

[14] Y. Nambu and G. Jona-Lasinio, Phys. Rev. 122, 345 (1961).

[15] M. Gell-Mann and Y. Ne'eman, The Eightfold Way (Benjamin, New York, 1964).
[16] J. Snygg, Clifford Algebra: A Computational Tool for Physicists (Oxford University Press, New York, 1997).

[17] J. Besprosvany and R. Romero, Nucl. and Part. Phys. Proc. 267-269, 199 (2015).

[18] M. Weinstein, Phys. Rev. D 8, 2511 (1973).

[19] P. Sikivie, L. Susskind, M. Voloshin, and V. Zakharov, Nucl. Phys. B173, 189 (1980).

[20] R. S. Chivukula, arXiv:hep-ph/9803219v2.

[21] Z. Z. Xing, H. Zhang, and S. Zhou, Phys. Rev. D 77, 113016 (2008); H. Fusaoka and Y. Koide, Phys. Rev. D 57, 3986 (1998).

[22] N. Cabibbo, Phys. Rev. Lett. 10, 531 (1963); M. Kobayashi and T. Maskawa, Prog. Theo. Phys. 49, 652 (1973).

[23] H. Fritzsch and Z. Z. Xing, Prog. Part. Nucl. Phys. 45, 1 (2000); C. D. Froggatt and H. B. Nielsen, Nucl. Phys. B147, 277 (1979).

[24] B. Pontecorvo, Sov. Phys. JETP 7, 172 (1958); Z. Maki, M. Nakagawa, and S. Sakata, Prog. Theor. Phys. 28, 870 (1962).

[25] J. Wess and J. Bagger, Supersymmetry and Supergravity (Princeton University Press, Princeton, NJ, 1983).

[26] E. Farhi and L. Susskind, Phys. Rep. 74, 277 (1981).

[27] W. A. Bardeen, C. T. Hill, and M. Lindner, Phys. Rev. D 41, 1647 (1990).

Correction: Minor subscript errors were found in Eq. (69) and have been fixed. 